February 2004 - NREL/TP-500-35109

\title{
Evaluation of RCAS Inflow Models for Wind Turbine Analysis
}

\author{
J. Tangler \\ G. Bir
}

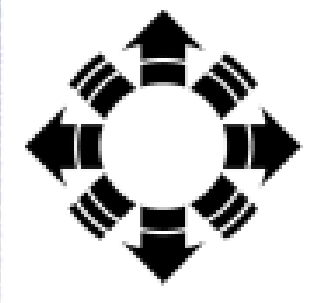

National Renewable Energy Laboratory

1617 Cole Boulevard

Golden, Colorado 80401-3393

NREL is a U.S. Department of Energy Laboratory Operated by Midwest Research Institute $\bullet$ Battelle

Contract No. DE-AC36-99-G010337 


\section{Evaluation of RCAS Inflow Models for Wind Turbine Analysis}

\section{J. Tangler}

G. Bir

Prepared under Task No. WER3 3110

\section{National Renewable Energy Laboratory}

1617 Cole Boulevard

Golden, Colorado 80401-3393

NREL is a U.S. Department of Energy Laboratory

Operated by Midwest Research Institute • Battelle

Contract No. DE-AC36-99-G010337 


\section{NOTICE}

This report was prepared as an account of work sponsored by an agency of the United States government. Neither the United States government nor any agency thereof, nor any of their employees, makes any warranty, express or implied, or assumes any legal liability or responsibility for the accuracy, completeness, or usefulness of any information, apparatus, product, or process disclosed, or represents that its use would not infringe privately owned rights. Reference herein to any specific commercial product, process, or service by trade name, trademark, manufacturer, or otherwise does not necessarily constitute or imply its endorsement, recommendation, or favoring by the United States government or any agency thereof. The views and opinions of authors expressed herein do not necessarily state or reflect those of the United States government or any agency thereof.

Available electronically at http://www.osti.gov/bridge

Available for a processing fee to U.S. Department of Energy and its contractors, in paper, from:

U.S. Department of Energy

Office of Scientific and Technical Information

P.O. Box 62

Oak Ridge, TN 37831-0062

phone: 865.576 .8401

fax: 865.576.5728

email: reports@adonis.osti.gov

Available for sale to the public, in paper, from:

U.S. Department of Commerce

National Technical Information Service

5285 Port Royal Road

Springfield, VA 22161

phone: 800.553.6847

fax: 703.605.6900

email: orders@ntis.fedworld.gov

online ordering: http://www.ntis.gov/ordering.htm 


\section{Contents}

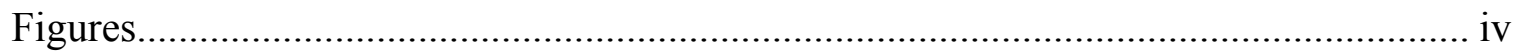

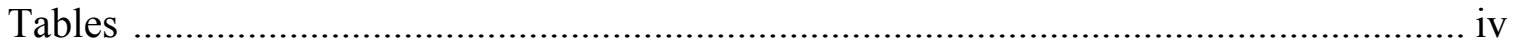

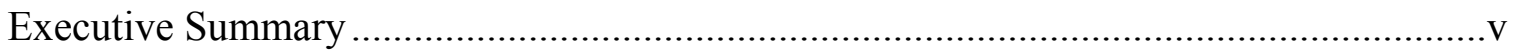

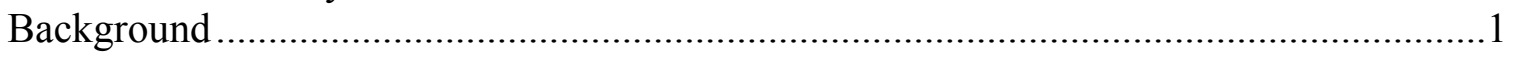

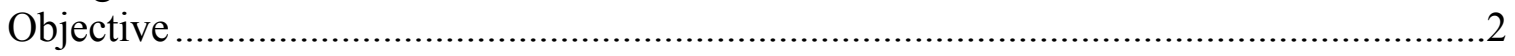

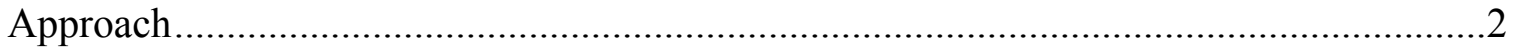

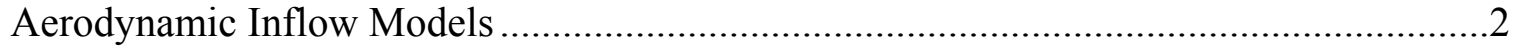

Blade Element Momentum Theory ................................................................... 3

Generalized Dynamic Wake Theory.................................................................... 3

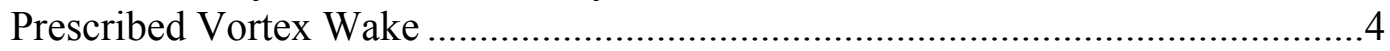

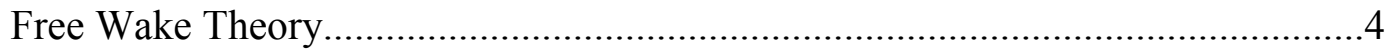

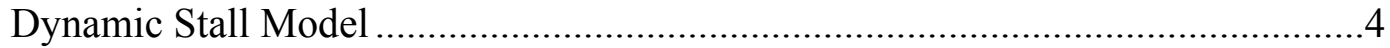

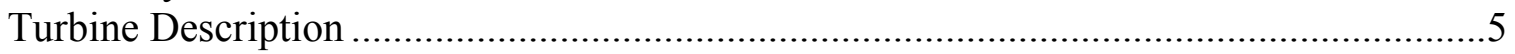

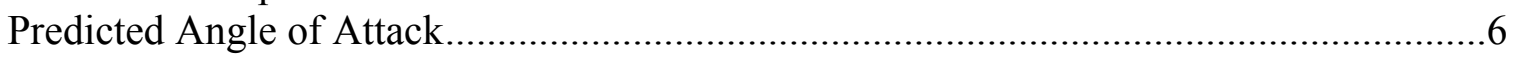

Steady State, Axis Symmetric Results ......................................................6

Quasi-Steady State, Yawed Flow Results..................................................

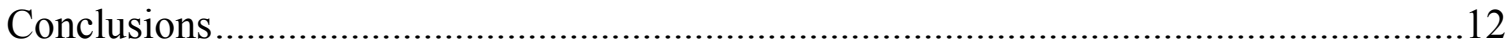

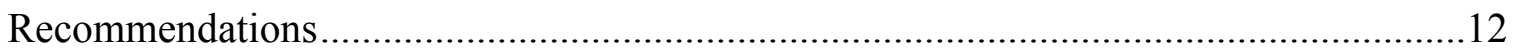

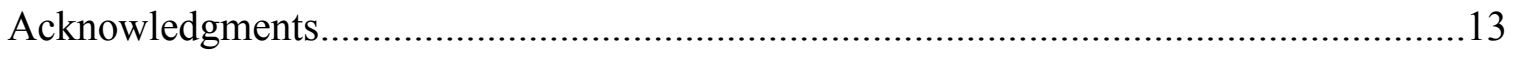

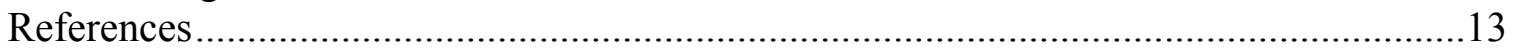

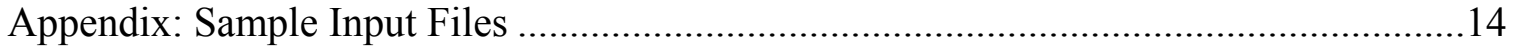

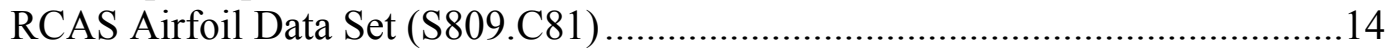

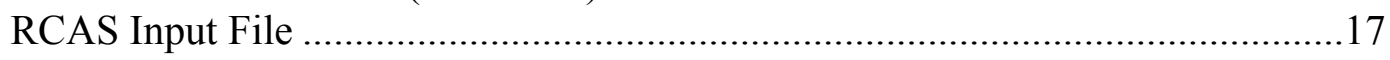

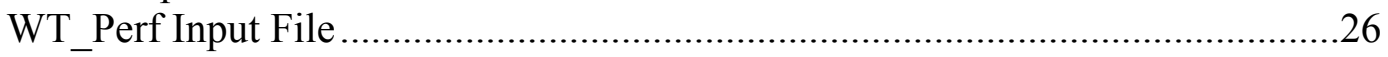

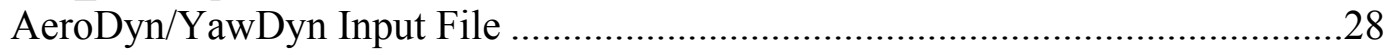

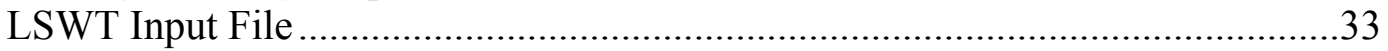




\section{Figures}

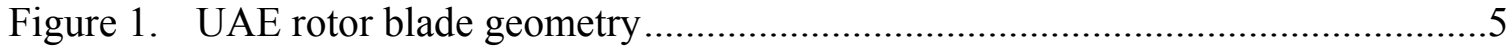

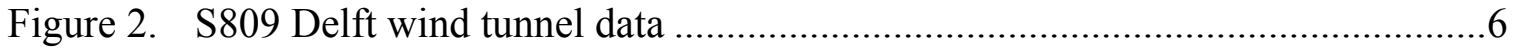

Figure 3. Effect of Prandtl tip/hub loss and induced swirl in WT Perf..........................6

Figure 4. Angle of attack comparison of WT_Perf, AeroDyn, and RCAS .....................7

Figure 5. Angle of attack comparison with generalized dynamic wake [4] ....................8

Figure 6. Angle of attack comparison for several wind speeds...................................

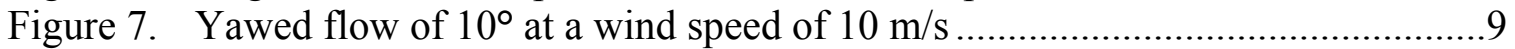

Figure 8. Yawed flow of $30^{\circ}$ at a wind speed of $10 \mathrm{~m} / \mathrm{s}$..........................................10

Figure 9. RCAS dynamic stall for $30^{\circ}$ yaw at a wind speed of $10 \mathrm{~m} / \mathrm{s}$........................10

Figure 10. AeroDyn dynamic stall for $30^{\circ}$ yaw at a wind speed of $10 \mathrm{~m} / \mathrm{s}$....................11

Figure 11. RCAS/AeroDyn dynamic stall for $30^{\circ}$ yaw at a wind speed of $10 \mathrm{~m} / \mathrm{s} \ldots \ldots \ldots . .11$

\section{Tables}

Table 1. Program and Inflow Models Used in This Study.............................................2

Table 2. Summary of Baseline Turbine Design Properties...............................................5 


\section{Executive Summary}

The finite element structural modeling in the Rotorcraft Comprehensive Analysis System (RCAS) provides a state-of-the-art approach to aeroelastic analysis. This, coupled with its ability to model all turbine components, results in a methodology that can simulate complex system interactions characteristic of large wind. In addition, RCAS is uniquely capable of modeling advanced control algorithms and the resulting dynamic responses.

The aerodynamic inflow models in RCAS have been formulated for helicopter analysis. These models needed to be evaluated for wind turbine analysis to determine whether they included features that accurately predict the inflow for steady state, quasi-steady state, and unsteady operating conditions of wind turbines. The aerodynamic inflow models include the generalized dynamic inflow model, the equilibrium-based blade element momentum (BEM) theory, a prescribed vortex wake, and a free vortex wake. The four inflow methods in RCAS are listed in order of increasing complexity and computational time. The inflow models in RCAS were evaluated by comparing their predicted angles of attack for steady and quasi steady operating conditions with other wind industry aerodynamic models such as WT_Perf (BEM), AeroDyn (BEM and generalized dynamic wake), and LSWT (lifting surface prescribed wake).

Momentum theory or the generalized dynamic-wake theory should be selected for most applications because both are simple and have rapid computational times. For unsteady inflow conditions the generalized dynamic wake inflow model is preferred because of its noniterative time domain formulation. The use of momentum theory in RCAS will depend on successful implementation of the Prandtl tip/hub loss model, induced swirl effects, and the Glauert approximation. Both the prescribed and free vortex wake options require exponential increases in computational time and use a basic lifting line blade representation. In unsteady inflow conditions these inflow models are less compatible with dynamic perturbations because of their discretized blade and wake vortex models. The prescribed and free wake vortex models are better suited for steady-state, axissymmetric performance prediction. However, for the prescribed and free wake vortex models, a lifting surface blade representation is preferred over a lifting line. 


\section{Background}

The Rotorcraft Comprehensive Analysis System (RCAS) is a state-of-the-art computational analysis system for modeling rotorcraft [1-4]. It can model a complete range of rotorcraft configurations in hover, forward-flight, and maneuvering flight conditions. RCAS can perform a variety of engineering calculations, including vehicle performance, aerodynamics and rotor loads, vehicle vibration, flight-control analysis, aeroelastic stability, flight dynamics, and flight simulation. Analysis features in RCAS include trim, maneuver, and stability.

Structural modeling in RCAS uses a finite element approach for the various rotorcraft components. A helicopter model can include a conventional or multi-rotor configuration along with the fuselage, engine/drivetrain model, and control system. This structural modeling provides the capability for accurate dynamic simulation that includes the interaction between all system components. The rotorcraft system structural modeling capability can be adapted to model wind turbine systems, which would include the rotor, generator/drivetrain, nacelle, tower, and control system. Because wind turbines are becoming larger and more dynamically active, a comprehensive system dynamic simulation is more critical.

Aerodynamic modeling in RCAS includes all rotorcraft system components such as rotors, wings, and fuselage. Two-dimensional airloads are used for blade and wing segments, three-dimensional airloads for the fuselage, and disc inflow models for the rotor. The resulting induced velocities and airloads are modeled separately for each component and collected in an aerodynamic library. RCAS includes four aerodynamic models that have been tailored for helicopter analysis to calculate the rotor's induced inflow. Aerodynamic inflow models include the generalized dynamic wake theory [5], blade element momentum (BEM), prescribed wake, and free wake. These inflow methods are listed in order of increasing computational time and complexity. The BeddoesLeishman dynamic stall model [6] can be used with all inflow models. The generalized dynamic wake theory or BEM should be selected for most applications because they are simple and have rapid computational times. Both the prescribed and free vortex wake options result in exponential increases in computational time and use the basic lifting line blade representation rather than a lifting surface.

The focus of this study was the aerodynamic modeling of the rotor in RCAS and assessing the suitability of the inflow models for predicting wind turbine blade loads and performance. Evaluation of the RCAS inflow models consisted of comparing their angleof-attack predictions to similar inflow models used in-house and in the wind industry. These models include WT_Perf [7], AeroDyn [8,9] and LSWT (Lifting-Surface Prescribed-Wake code) [10]. Both WT_Perf and AeroDyn have a BEM inflow model to predict rotor performance. In addition, AeroDyn includes the generalized dynamic wake inflow model and the Beddoes-Leishman dynamic stall model. Both codes can be used to predict steady state axis-symmetric performance and quasi steady state, yawed rotor performance. RCAS and AeroDyn, with the generalized dynamic wake theory can be used for unsteady performance prediction. The LSWT performance prediction code uses a prescribed, expanding-wake, inflow model. The code uses a lifting surface blade 
representation for blade geometry optimization and can also be used to predict steadystate, axis-symmetric performance and quasi-steady-state, yawed rotor performance. The comparison in this report included exercising all the inflow options in RCAS relative to those in NREL's in-house performance prediction codes WT_Perf, AeroDyn, and LSWT. This was done using the UAE (unsteady aerodynamic experiment) two bladed, rotor geometry [11] and S809 [12] airfoil characteristics.

\section{Objective}

The objective of this study was to evaluate the aerodynamic inflow options in RCAS. To accomplish this objective, predicted angle of attack distributions obtained from RCAS were compared with industry-accepted aerodynamic codes that included WT_Perf, AeroDyn, and LSWT. The project scope was limited to axis-symmetric and yawed-flow, steady state operation with no structural degrees of freedom.

\section{Approach}

The UAE Phase VI wind turbine was selected for this code comparison. The upwind configuration of this two-bladed, horizontal-axis turbine has been the subject of several past studies. Consequently, input data for this turbine were available and results from this study will provide a baseline for future comparisons with UAE test data from the NASA Ames $80-\mathrm{ft}$ by $120-\mathrm{ft}(24.5-\mathrm{m}$ by $36.5-\mathrm{m})$ wind tunnel. This $10-\mathrm{m}(33-\mathrm{ft})$ rotor has tapered, twisted blades that use the NREL S809 airfoil profile from blade root to blade tip. S809 2-D airfoil data, from the Delft low turbulence wind tunnel, was used for calculating angle of attack distributions in RCAS, WT_Perf, AeroDyn, and LSWT. Operating cases included steady state axis-symmetric operation and quasi-steady state yawed flow operation.

\section{Aerodynamic Inflow Models}

RCAS, WT_Perf, and AeroDyn all employ the BEM inflow option for modeling wind turbine aerodynamics (see Table 1). In addition, RCAS and AeroDyn include a dynamic inflow option that minimizes computational time. For stall and post-stall calculations the prescribed wake inflow approach in LSWT and RCAS have the potential to more accurately predict the angle of attack. The free wake option in RCAS provides further flexibility to accommodate more inflow conditions at the expense of excessive computer time. Detailed descriptions of the theories each code employs can be found in their user and theory manuals.

Table 1: Program and Inflow Models Used in this Study

\begin{tabular}{|l|l|}
\hline RCAS 1.9.5a & BEM, Dynamic Inflow, Prescribed Wake, Free Wake \\
\hline WT_Perf 2.2 & BEM \\
\hline AeroDyn 12.50 & BEM, Dynamic Inflow \\
\hline LSWT 1.2 & Prescribed Wake \\
\hline
\end{tabular}




\section{Blade Element Momentum Theory}

Below stall, BEM theory predicts reasonable angle-of-attack distributions for steadystate, axis-symmetric inflow conditions. Under stalled airfoil conditions, normally at high wind speeds, BEM over predicts the angle-of-attack distribution [13]. The over prediction is largely the result of two simplifying assumptions inherent in BEM. The "uniform inflow around the annulus" assumption leads to an over prediction of the angle of attack with the onset of stalled rotor conditions. The "no interaction between annuluses" assumption does not recognize the strong induced effect of trailing vorticity on the angle of attack distribution and resulting load distribution.

For helicopter analysis, RCAS uses an effective-radius tip-loss model with no hub loss model. Typically, an effective radius input of 0.98 is used for helicopters, which means the blade radius is reduced by $2 \%$. For wind turbines, the shorter radius from this tip loss model results in a larger blade pitch angle to achieve the designated thrust and power. Because the effective radius tip loss model has little effect on the blade load distribution it is not appropriate for wind turbine calculations. The Prandtl tip and hub loss models favorably decrease the tip and root loading and should be implemented in RCAS for use with the BEM inflow option.

RCAS was formulated primarily for helicopter forward flight calculations, in which the axial induction factor is important and the swirl induction factor is insignificant. However, for wind turbine calculations the induced swirl is significant and results in a lower angle of attack distribution toward the blade root region. The induced swirl should be implemented in RCAS to improve accuracy and to provide results comparable with other wind turbine codes that include the induced swirl.

For wind turbines, the Glauert empirical approximation improves accuracy at low wind speeds, high tip speed ratios, and high axial induction factors. The Glauert empirical approximation (also known as the windmill brake state model) calculates higher power coefficients than the classical momentum method at non-optimum pitch angles toward feather. This empirical approximation should be included in RCAS.

\section{Generalized Dynamic Wake Theory}

The generalized dynamic wake model is based on incompressible, potential flow, and small disturbance theory. This inviscid, non-interative, three-dimensional unsteady induced-flow theory is based on the unsteady theory of Peters and He [4]. The time domain model uses acceleration potential with a skewed cylindrical wake. This method calculates only the dynamic inflow perpendicular to the rotor disc as a function of the disc loading and air mass dynamic force. Wake induced swirl in the rotor plane, which is not important to helicopter analysis, is not calculated. The method implicitly includes Theodorsen and Loewy induced inflow effects from the shed wake as well as PrandtlGoldstein tip losses caused by the trailing wake. This inflow method is useful in analyzing skewed wake effects when operating off the wind axis. For helicopters, the method provides good correlation of the inflow relative to measurements, except near the blade tip. For yawed-flow calculations the method approximates the time lag between the initiation of a load disturbance and when it is felt at some other point in the flow field. Of 
all the inflow models it is best suited for aeroelasticity analysis, aeroelasticity tailoring, and higher harmonic control. It can also be applied to investigate coupled blade flap, lead-lag, and torsional stability.

\section{Prescribed Vortex Wake Theory}

Simplifying assumptions associated with blade element momentum and the generalized dynamic wake theories are largely eliminated through the use of a prescribed vortex wake. The method is better suited for axis-symmetric, blade geometry and performance optimization than either BEM or generalized dynamic wake theory, since it includes wake distortion and wake rollup. However, vortex wake inflow methods lend themselves to steady and quasi-steady analysis but are not well suited for unsteady aeroelasticity analysis. This drawback relates to the discretization of both the blade and wake vortex model, which tends to be less compatible with dynamic perturbations. The wake inflow model would likely need five inflow points per half cycle for the highest frequency of interest. Such a fine inflow resolution would lead to excessive computer time and frequent numerical instabilities. The RCAS prescribed wake model is based on helicopter contracting, prescribed wake equations that are limited to a constant diameter wake equal to the rotor diameter. Expanding wake prescribed equations, similar to those used in LSWT, are needed for this option to be viable for wind turbine analysis. In addition, the RCAS prescribed wake is used with a lifting line blade representation, whereas the expanding prescribed wake equations in LSWT are used with a lifting surface blade representation that includes induced effects for as many as five chordwise panels. Lacking the correct wind turbine prescribed wake equations, the RCAS prescribed wake model resulted in excessively high angle of attack predictions.

\section{Free Wake Theory}

A free wake inflow model is unencumbered by the use of prescribed wake equations and can accommodate a broader range of operating conditions. Several free wake formulations differ primarily in their numerical methods. The Leishman and Bhagwat free-vortex wake is the newest free wake option to be used in RCAS. For this wake model, the tip region trailing vorticity is truncated into a discrete tip vortex $15^{\circ}-45^{\circ}$ behind the blade. The free wake extends one and a half radii below the blade. The bottom of free wake is stabilized by evolutions of prescribed wake resulting from previous free wake iterations. Like the prescribed wake, the free wake is not well suited for unsteady aeroelasticity analysis because of wake discretization. Attempts to run the free wake for the UAE wind turbine rotor were not successful. The input data set included a large number of wake geometry input parameters that will require some trial and error to find a suitable combination.

\section{Dynamic Stall Model}

Dynamic stall behavior is characterized using the Beddoes-Leishman dynamic stall model with any of the RCAS inflow models. AeroDyn also uses the same dynamic stall model. The dynamic stall model is most compatible with the time domain based, generalized dynamic wake inflow model in RCAS and AeroDyn. In RCAS, the dynamic stall model is airfoil dependent and numerous airfoil specific input based to 2-D unsteady tests are required. Airfoil specific inputs in RCAS are defined only for the symmetric NACA 0012 airfoil and the SC1095 cambered airfoil. Only the cambered airfoil should 
be used in wind turbine analysis. In AeroDyn no airfoil inputs are required since the dynamic stall model is hard wired for a generic cambered airfoil.

\section{Turbine Description}

The UAE turbine geometry and aerodynamic information were obtained for the Phase VI UAE Rotor [10]. A summary of the turbine design properties for this two-bladed, upwind, horizontal-axis wind turbine appears in Table 2.

Table 2: Summary of Baseline Turbine Design Properties

\begin{tabular}{|l|l|}
\hline Rotor Diameter & $10.06 \mathrm{~m}$ \\
\hline Hub Height & $12.2 \mathrm{~m}$ \\
\hline Rotor Precone & $0^{\circ}$ \\
\hline Shaft Tilt & $0^{\circ}$ \\
\hline Rotor Speed & $72 \mathrm{rpm}$ \\
\hline Tip Speed Ratio for Maximum Power Coefficient & 7.0 \\
\hline
\end{tabular}

The UAE rotor blade geometry is shown in Figure 1. The blade uses the S809 airfoil from root to tip. Performance characteristics for the S809 airfoil, acquired in the Delft wind tunnel, are shown in Figure 2. For simplicity, the lift and drag data for a Reynolds number of 1,000,000 were used from blade root to tip in WT_Perf, AeroDyn, LSWT, and RCAS.

The airfoil data input format for RCAS is derived from a Bell Helicopter's dynamics code, "C81", which was tailored for helicopter analysis. Each angle of attack in the data table is listed for multiple Mach numbers. For each Mach number, lift, drag, and moment coefficient are given. For wind turbines, compressibility effects are negligible and the airfoil characteristics can be held constant with Mach number. An example of a wind turbine airfoil input data set (S809.C81) with the S809 airfoil is shown in the Appendix. A simpler format is desired for wind turbine analysis.

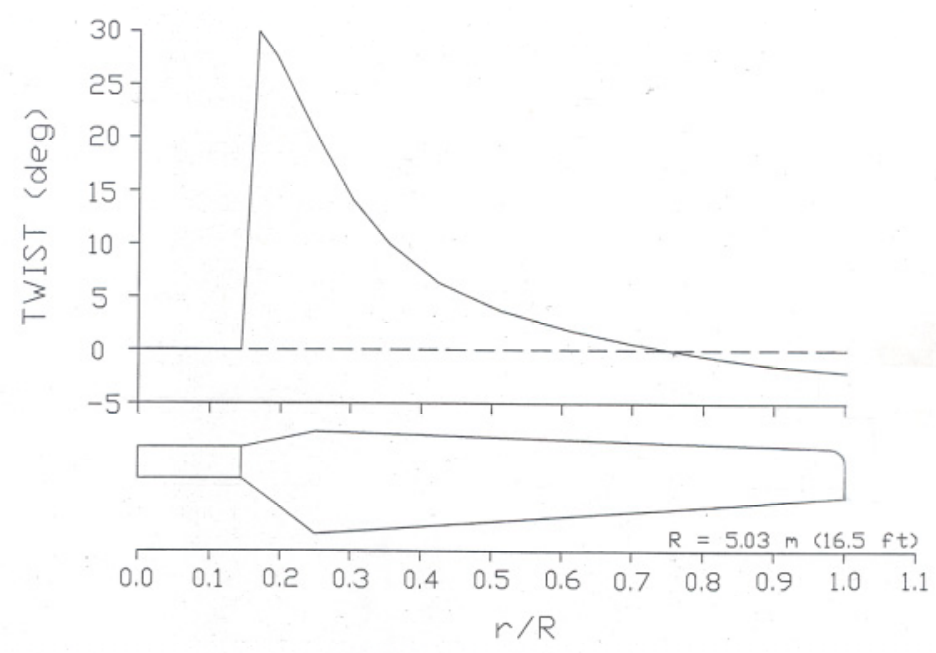

Figure 1. UAE rotor blade geometry. 


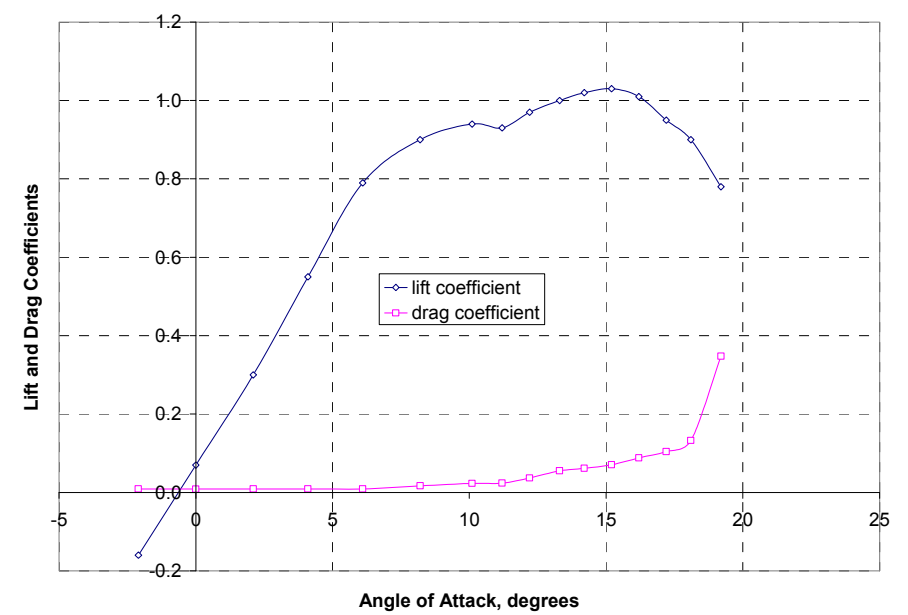

Figure 2. S809 Delft wind tunnel data.

\section{Predicted Angle of Attack}

\section{Steady State Axis Symmetric Results}

The BEM inflow option in RCAS neglects several options - induced swirl, the Prandtl tip loss option, and the Glauert approximation-important to wind turbine analysis. NREL's in-house BEM code, WT Perf was used to illustrate the influence of the induced swirl and Prandtl tip loss option on the predicted angle of attack. Figure 3 shows the predicted angle of attack for the UAE two-bladed rotor at $7 \mathrm{~m} / \mathrm{s}$ when no stall is present along the blade. Induced swirl, which adds to the local rotational speed, is greatest over the inboard portion of the blade and decreases with radius. Consequently, the inclusion of swirl decreases the angle of attack inboard by about $1^{\circ}$ with almost no effect toward the tip. The Prandtl tip loss reduced the angle of attack by about $2^{\circ}$ toward the tip. The Prandtl hub loss results in a fraction of a degree reduction toward the blade root. The Glauert approximation has little influence on the angle of attack distribution for optimum blade pitch.

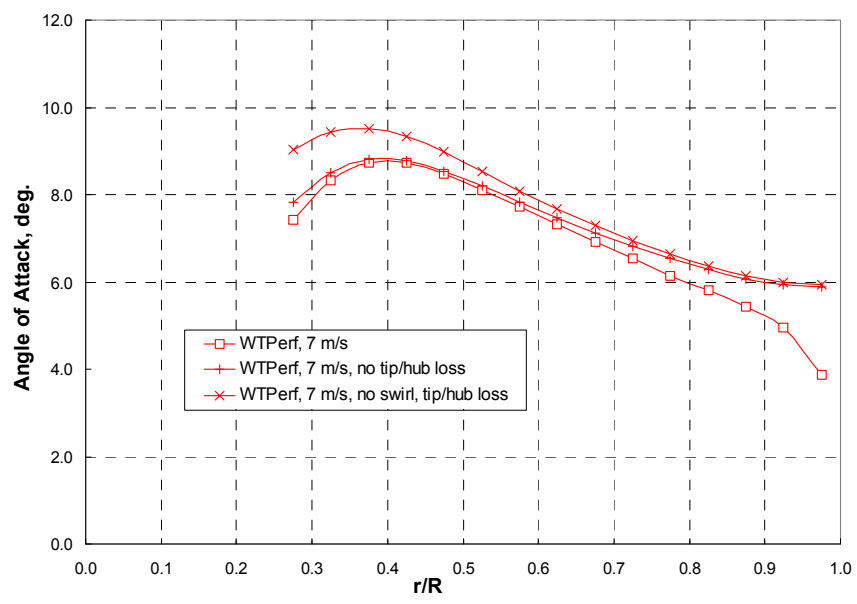

Figure 3. Effect of Prandtl tip/hub loss and induced swirl in WT_Perf. 


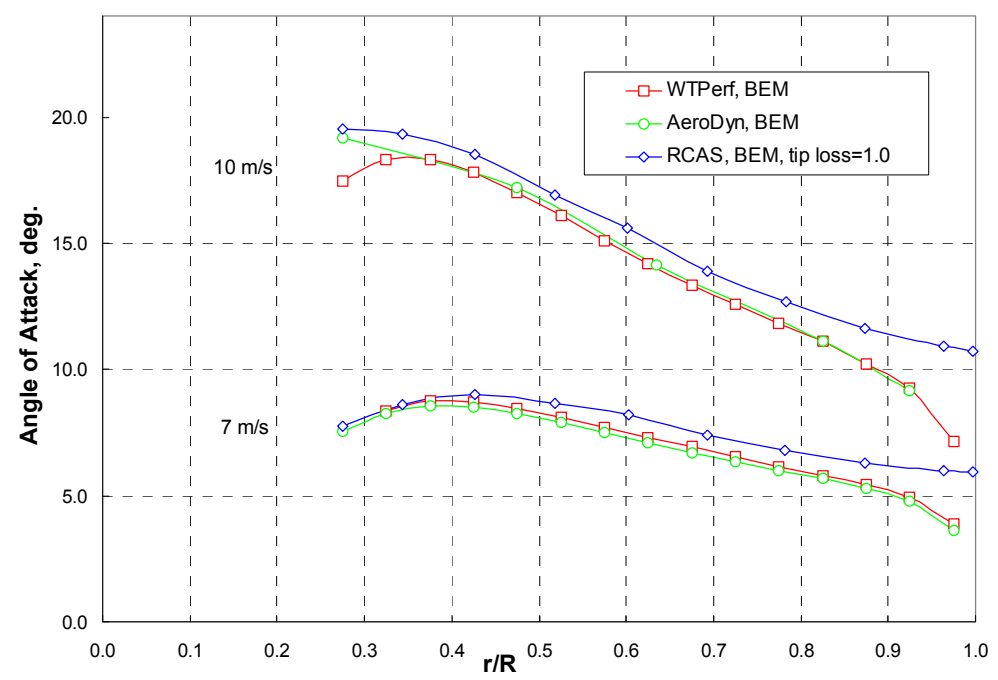

Figure 4. Angle of attack comparison of WT_Perf, AeroDyn, and RCAS.

The BEM inflow option was used to show a comparison of the predicted angle of attack for WT_Perf, AeroDyn, and RCAS for wind speeds of 7 and $10 \mathrm{~m} / \mathrm{s}$ (Figure 4). Close agreement is seen for WT_Perf and AeroDyn, both of which include the induced swirl, Prandtl tip loss, and Glauert approximation. WT_Perf also includes the Prandtl hub loss that is omitted in this version of AeroDyn. The higher blade root angle of attack is attributed to this omission. For both wind speeds, RCAS predicts noticeably higher angle of attack distributions by not including these inflow options.

The dynamic wake inflow option [4] in RCAS provided better agreement with WT_Perf and LSWT over the outboard part of the blade (see Figure 5). For comparison, the RCAS BEM inflow option, with the over-predicted angle of attack is also shown. Both the BEM method WT_Perf and the prescribed wake method LSWT are in close agreement at 7 $\mathrm{m} / \mathrm{s}$, except for the root region where LSWT has a lower angle of attack as a result of greater induced root inflow. The dynamic inflow option of RCAS over predicts the tip and hub region angle of attack even though the method implicitly includes the PrandtlGoldstein tip losses. Also unusual is the lower angle of attack (around 50\% radius) when induced swirl effects are not included in the dynamic wake inflow option.

Further comparison of the RCAS dynamic wake inflow option is shown in Figure 6 for wind speeds of 5,7 , and $10 \mathrm{~m} / \mathrm{s}$. The dynamic inflow option tends to under predict the angle of attack distribution with increasing wind speeds, except for the immediate tip and hub region. Also, WT_Perf over predicts the angle of attack with increasing wind speed as a result of the uniform inflow assumption around the annulus and the assumption of no interaction between annuli. 


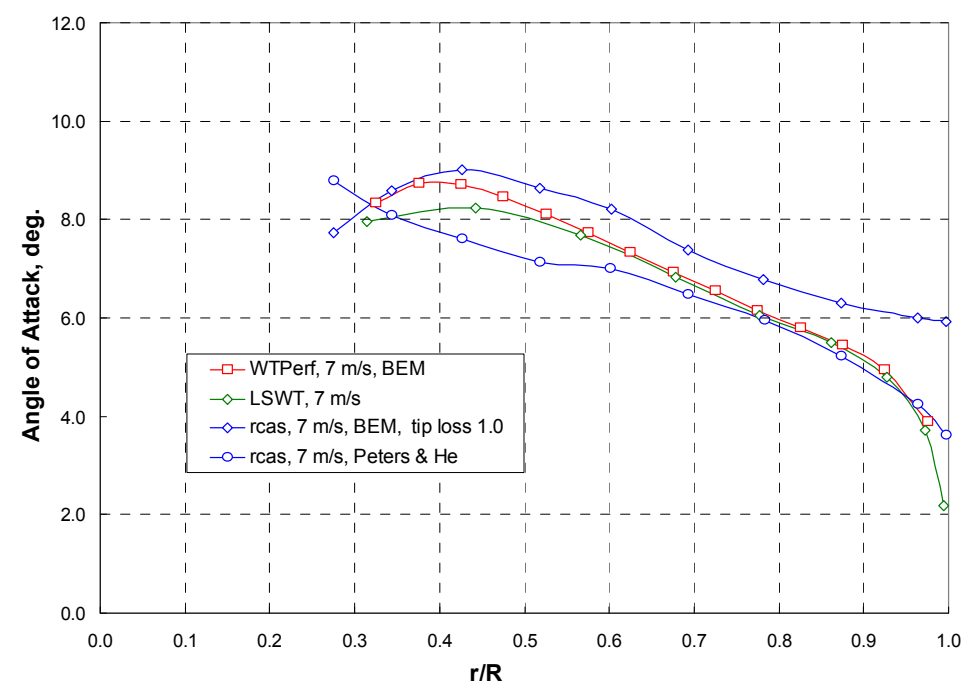

Figure 5. Angle of attack comparison with generalized dynamic wake [4].

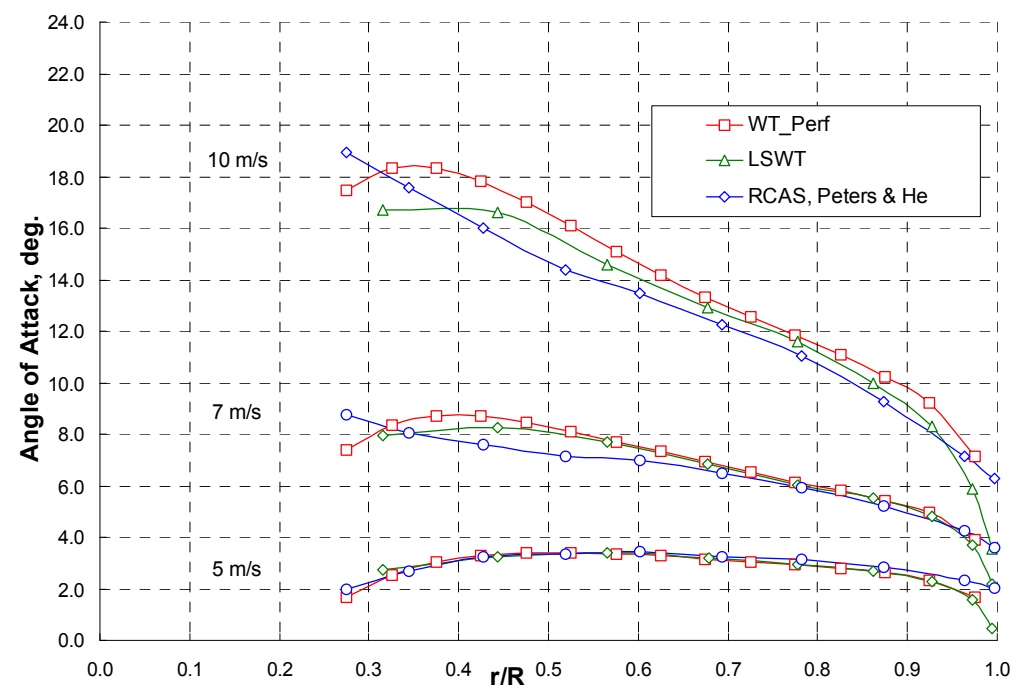

Figure 6. Angle of attack comparison for several wind speeds.

\section{Quasi-Steady State Results}

Angle of attack predictions at $10 \mathrm{~m} / \mathrm{s}$ were generated for yaw angles of $10^{\circ}$ and $30^{\circ}$ at three blade radial stations $(\mathrm{r} / \mathrm{R}=0.42,0.87$, and 0.97$)$. The dynamic wake inflow model was used for RCAS because it is more accurate than the other inflow models. AeroDynAeroDyn comparisons are also provided with its dynamic wake inflow model. The comparisons show angle of attack versus blade azimuth. Zero blade azimuth is with the blade at the top of the rotor disc advancing into the wind. The advancing blade half of the disc would be $270^{\circ}-90^{\circ}$; the retreating half would be $90^{\circ}-270^{\circ}$.

For a yaw angle of $10^{\circ}$, a comparison of angle of attack distribution versus blade azimuth is shown in Figure 7. At $\mathrm{r} / \mathrm{R}=0.97$, RCAS, AeroDyn, and LSWT all agree with 


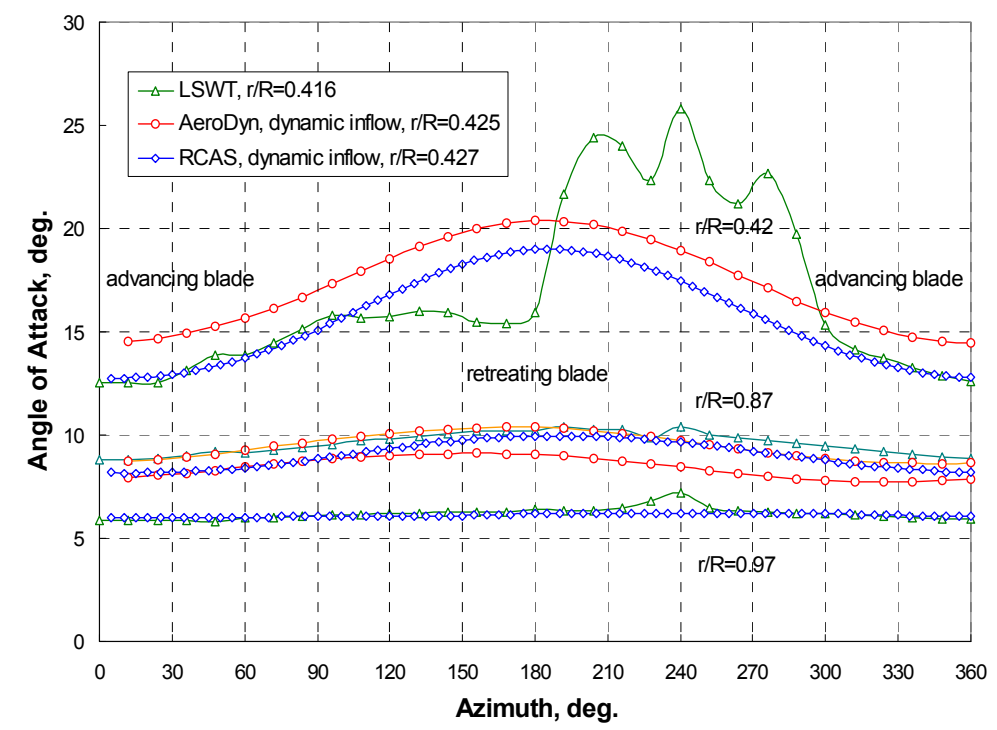

Figure 7. Yawed flow of $10^{\circ}$ at a wind speed of $10 \mathrm{~m} / \mathrm{s}$.

an angle of attack slightly higher than $6^{\circ}$. Moving inboard to $\mathrm{r} / \mathrm{R}=0.87$, the angle of attack phasing is somewhat different even though both RCAS and AeroDyn use a dynamic inflow formulation. LSWT appears to be in better agreement with RCAS. Also in the root region RCAS and LSWT are in good agreement except for a large blade/vortex interaction on the retreating blade. In the root region AeroDyn predicts an angle of attack several degrees higher than the other two methods. For higher yaw angles LSWT would not converge because of numerical problems resulting from a lack of a finite vortex core model that would limit extremely high induced velocities resulting from blade/vortex interactions.

At a yaw angle of $30^{\circ}$, a similar comparison of RCAS and AeroDyn was done with the dynamic wake inflow models (see Figure 8). Also shown for comparison is a WT_Perf prediction with BEM inflow. Toward the tip, WT_Perf predicts a lower angle of attack. The dynamic inflow model in RCAS and AeroDyn results in an angle of attack several degrees higher. Again, RCAS and AeroDyn exhibit an unexplained magnitude and phasing shift of the angle of attack relative to one another. At $r / R=0.87$ the differences between the three methods are much smaller. Blade root region differences become more significant; most noticeable are the previously mentioned angle of attack phase shifts between RCAS and AeroDyn. The angle of attack distribution of AeroDyn is slightly less symmetrical or biased to the right. 


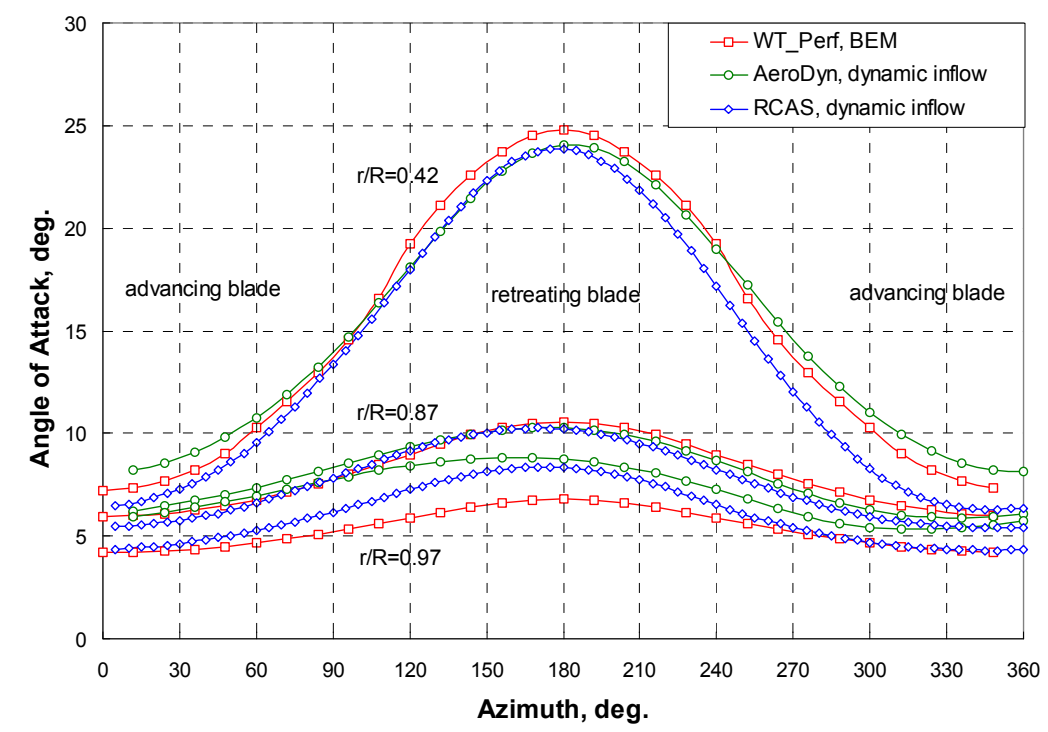

Figure 8. Yawed flow of $30^{\circ}$ at a wind speed of $10 \mathrm{~m} / \mathrm{s}$.

The dynamic stall option in RCAS was turned on for comparative purposes. Dynamic stall, option 5, was used and is considered to be the most rigorous option. The dynamic stall model requires airfoil specific inputs for the S809 airfoil. Similar airfoil inputs from the program's cambered SC 1098 airfoil, used on the Blackhawk helicopter, were chosen since S809 inputs were not available. With dynamic stall turned on, the primary change is a decrease in angle of attack and a phase shift to the right (see Figure 9). With the dynamic stall model turned on in AeroDyn, a similar change is observed (Figure 10). Dynamic stall models in both AeroDyn and RCAS are based on the Leishmann-Beddoes method, but AeroDyn has the airfoil inputs hardwired with no user-selected inputs. An overlay of the dynamic stall predicted angle of attack distributions for RCAS and AeroDyn is shown in Figure 11. AeroDyn

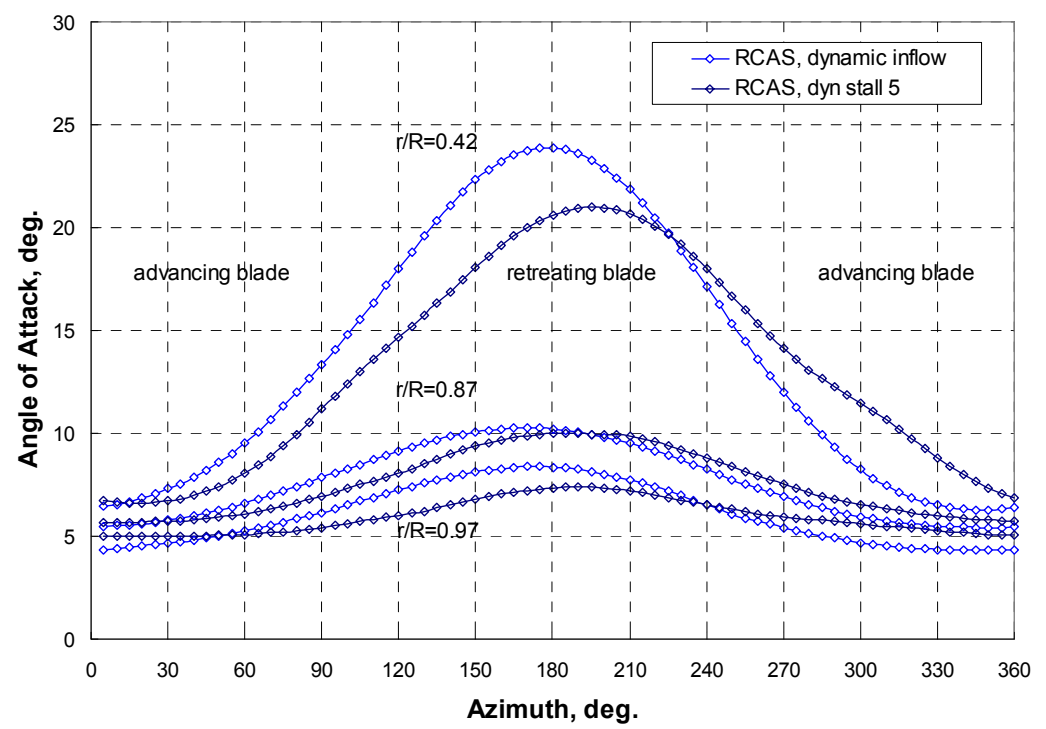

Figure 9. RCAS dynamic stall for 30 degrees yaw at a wind speed of $10 \mathrm{~m} / \mathrm{s}$. 
predicts a higher angle of attack peak than RCAS. For AeroDyn, toward the blade root, the angle of attack distribution is several degrees greater from a blade azimuth of $180^{\circ}-$ $90^{\circ}$. This increase may not be related to the difference in dynamic stall models but to shift without the dynamic stall model.

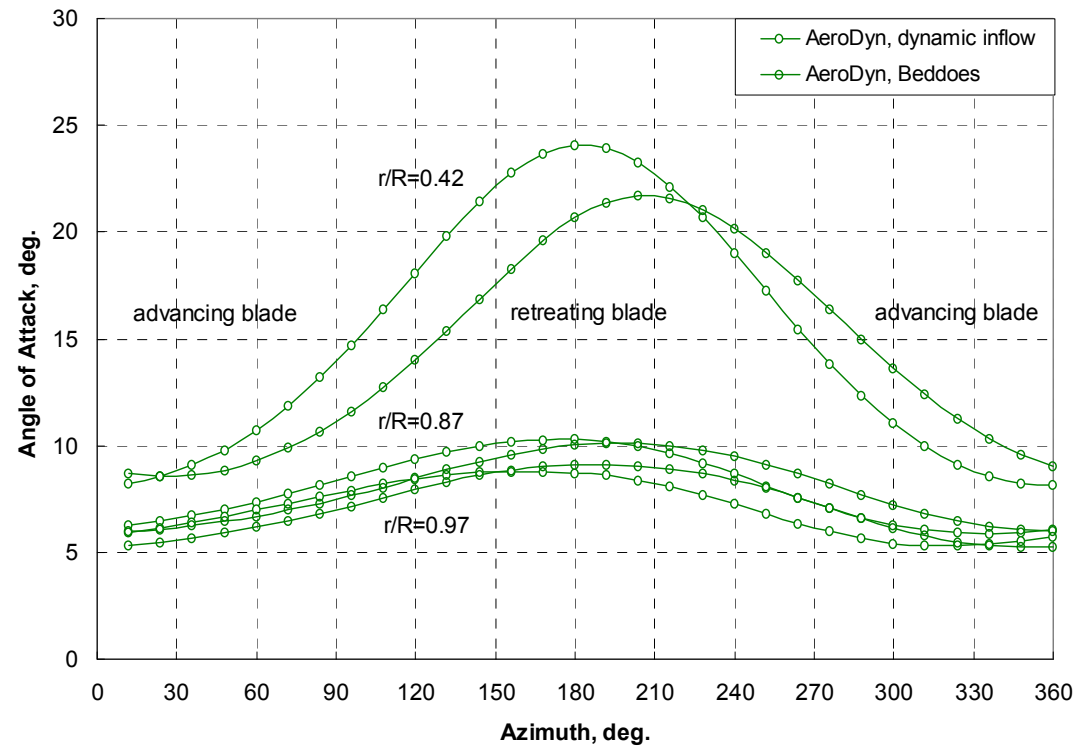

Figure 10. AeroDyn dynamic stall for $30^{\circ}$ yaw at a wind speed of $10 \mathrm{~m} / \mathrm{s}$.

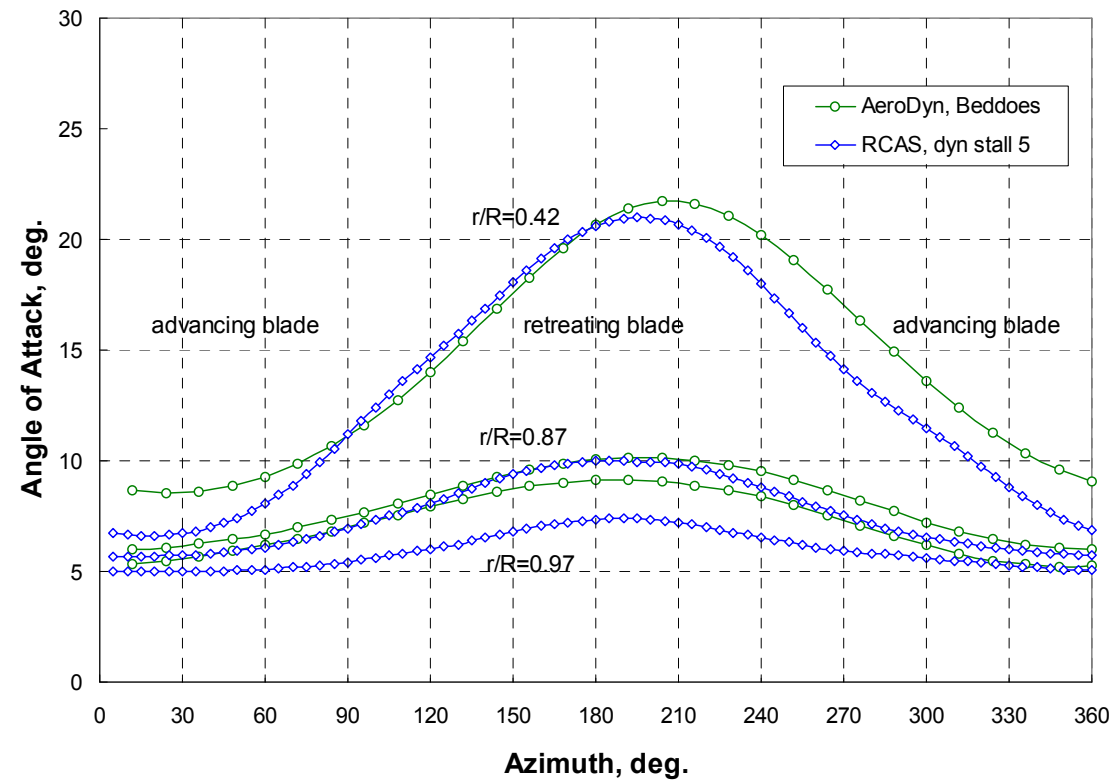

Figure 11. RCAS/AeroDyn dynamic stall, $30^{\circ}$ yaw at a wind speed of $10 \mathrm{~m} / \mathrm{s}$. 


\section{Conclusions}

Evaluation of the four aerodynamic inflow models in RCAS resulted in the following findings. BEM, which is used for most codes, lacks several features-Prandtl tip and hub loss model, induced swirl calculations, and the Glauert approximation for use at high thrust coefficients - needed for wind turbine analysis. Without these features, the BEM inflow option in RCAS over predicts the angle of attack distribution. The generalized dynamic wake inflow option in RCAS provided a reasonable prediction of the angle of attack distribution. Inherent in this model is a Prandtl-Goldstein based tip loss correction. Even with the tip loss correction, the local tip angle of attack is over predicted relative to BEM and LSWT theories. The wake inflow option does not include induced swirl effects. This does not appear to be a significant impediment, since the angle of attack distribution toward the blade root where the angle is reduced by swirl is already under predicted. Being a time domain, noniterative inflow model, the generalized dynamic wake is the only inflow model practical for unsteady inflow and aeroelasticity calculations. This model will also provide the lowest computational time of the four inflow models. The prescribed and free-wake inflow options are not as well suited for unsteady flow conditions because both the blade and wake vortex model, which are less compatible with dynamic perturbations, are discretized. The prescribed and free-wake inflow options are better used for axis-symmetric performance prediction and blade geometry optimization. They both use a basic lifting-line blade representation and the prescribed wake method lacks equations to simulate an expanding wind turbine wake. For axis-symmetric performance predictions, the LSWT is currently a better choice since it is tailored for wind turbine analysis.

\section{Recommendations}

Once the Prandtl tip/hub loss model, induced swirl, and with the Glauert approximation are implemented in RCAS, further validation of the BEM inflow option is needed to verify the angle of attack distributions. A more conventional airfoil data input format is needed in RCAS for wind turbine analysis. A wind turbine user's manual with examples is needed to minimize confusion. Wind turbine prescribed wake equations are necessary for the prescribed-wake inflow option. The free-wake inflow option should be further explored to determine what combinations of wake parameters are suitable for wind turbine analysis. A dynamic stall input file is needed for a generic wind turbine airfoil.

Currently the lack of a comprehensive wind turbine user's manual makes RCAS difficult to use and results in a long and expensive learning curve. A wind turbine user's manual should include several well-documented wind turbine examples along with their input and output files.

In AeroDyn, for yawed flow conditions, the angle of attack asymmetry versus blade azimuth relative to RCAS should be investigated. In LSWT, for yawed flow conditions, a vortex core model is needed to limit the maximum induced velocity generated by a vortex filament. This should be complemented with a dynamic stall subroutine. Finally, predicted results should be compared with actual UAE data for the operating conditions investigated in this report. 


\section{Acknowledgments}

The authors would like to thank Hossein Saberi from ART, Inc. for the technical support he provided on the inner workings of RCAS. Additional thanks go to Marshall Buhl of NREL for his computer support and to Mike Robinson of NREL for his guidance and funding of this study.

\section{References}

[1] Anon. RCAS Theory Manual, Version 2.0, United States (US) Army Aviation and Missile COMmand/AeroFlightDynamics Directorate (USAAMCOM/AFDD) Technical Report (TR) 02-A-005, USAAMCOM/AFDD TR 02-A-005, U.S. Army Aviation and Missile Command, Moffett Field, CA, June 2002.

[2] Anon. RCAS User's Manual, Version 2.0, United States (US) Army Aviation and Missile COMmand/AeroFlightDynamics Directorate (USAAMCOM/AFDD) Technical Report (TR) 02-A-006, USAAMCOM/AFDD TR 02-A-006. Moffett Field, CA: U.S. Army Aviation and Missile Command, June 2002.

[3] Anon. RCAS Applications Manual, Version 2.0, United States (US) Army Aviation and Missile COMmand/AeroFlightDynamics Directorate (USAAMCOM/AFDD) Technical Report (TR) TR 02-A-007. Moffett Field, CA: U.S. Army Aviation and Missile Command, June 2002.

[4] Jonkman, J. and Cotrell, J. "A Demonstration of the ability of RCAS to Model Wind Turbines," NREL/TP-500-34632, August 2003.

[5] Peters, D.A. and He, C. "Correlation of Measured Induced Velocities with a FiniteState Wake Model," Journal of the American Helicopter Society, Vol. 36(3), July 1991.

[6] Leishman, J.G. and Beddoes, T.S. "A Semi-Empirical Model for Dynamic Stall," Journal of the American Helicopter Society, July 1989.

[7] Buhl Jr., M.L.; Wright, A.D.; and Tangler, J.L. "Wind Turbine Design Codes: A Preliminary Comparison of the Aerodynamics." Prepared for the $17^{\text {th }}$ American Society of Mechanical Engineers (ASME) Wind Energy Symposium, January 12-15, 1998, Reno, Nevada. NREL/CP-500-23975. Golden, CO: National Renewable Energy Laboratory, December 1997.

[8] Laino, D.J. and Hansen, A.C. User's Guide to the Wind Turbine Dynamics Computer Programs YawDyn, Windward Engineering, January 2003.

[9] Laino, D.J. and Hansen, A.C. User's Guide to the Wind Turbine Aerodynamics Computer Software AeroDyn, Windward Engineering, December 2002. 
[10] Kocurek, D, "Lifting Surface Performance Analysis for Horizontal Axis Wind Turbines,” SERI/STR-217-3163, 1987.

[11] Hand, M.M.; Simms D.A.; Fingersh, L.J.; Jager, D.W.; Cotrell, J.R.; Schreck, S.; and Larwood, S.M. Unsteady Aerodynamics Experiment Phase VI: Wind Tunnel Test Configurations and Available Data Campaigns. NREL/TP-500-29955. Golden, CO: National Renewable Energy Laboratory, December 2001.

[12] Somers, D.M., Design and Experimental Results for the S809 Airfoil, NREL/SR440-6918. Golden, CO: National Renewable Energy Laboratory, 1997.

[13] Tangler, J.L., "The Nebulous Art of Using Wind Tunnel Airfoil Data for Predicting Rotor Performance," Wind Energy, Vol. 5, No. 2/3, April-September 2002.

\section{Appendix: Sample Input Files}

\section{RCAS Airfoil Data Set (S809.C81)}

$\begin{array}{llllllll}180 . & 0 . & 0 . & 0 . & 0 . & 0 . & 0 . & 0 . \\ -172.5 & .78 & .78 & .78 & .78 & .78 & .78 & .78 \\ -161 . & .62 & .62 & .62 & .62 & .62 & .62 & .62 \\ -147 . & 1 . & 1 . & 1 . & 1 . & 1 . & 1 . & 1 . \\ -129 . & 1 . & 1 . & 1 . & 1 . & 1 . & 1 . & 1 . \\ -49 . & -1.18 & -1.18 & -1.18 & -1.18 & -1.18 & -1.18 & -1.18 \\ -39 . & -1.18 & -1.18 & -1.18 & -1.18 & -1.18 & -1.18 & -1.18 \\ -21 . & -.8 & -.8 & -.81 & -.83 & -.85 & -.85 & -.85 \\ -16.5 & -1.007 & -1.007 & -.944 & -.96 & -.965 & -.965 & -.965 \\ -15 . & -1.19 & -1.19 & -1.09 & -1.055 & -.99 & -.98 & -.98 \\ -14 . & -1.333 & -1.333 & -1.22 & -1.096 & -1 . & -.97 & -.97 \\ -13 . & -1.334 & -1.334 & -1.28 & -1.12 & -1 . & -.96 & -.96 \\ -12 . & -1.255 & -1.255 & -1.26 & -1.13 & -1 . & -.947 & -.94 \\ -11 . & -1.161 & -1.161 & -1.19 & -1.12 & -.994 & -.93 & -.923 \\ -10 . & -1.055 & -1.055 & -1.01 & -1.082 & -.985 & -.91 & -.90 \\ -8 . & -.844 & -.844 & -.88 & -.907 & -.922 & -.87 & -.84 \\ -6 . & -.633 & -.633 & -.66 & -.684 & -.741 & -.77 & -.75 \\ -4.1 & -.4 & -.4 & -.4 & -.4 & -.4 & -.4 & -.4 \\ -2.1 & -.16 & -.16 & -.16 & -.16 & -.16 & -.16 & -.16 \\ 0 . & 0.07 & 0.07 & 0.07 & 0.07 & 0.07 & 0.07 & 0.07 \\ 2.1 & .30 & .30 & .30 & .30 & .30 & .30 & .30 \\ 4.1 & .55 & .55 & .55 & .55 & .55 & .55 & .55 \\ 6.1 & .79 & .79 & .79 & .79 & .79 & .79 & .79 \\ 8.2 & .90 & .90 & .90 & .90 & .90 & .90 & .90 \\ 10.1 & .94 & .94 & .94 & .94 & .94 & .94 & .94 \\ 11.2 & .93 & .93 & .93 & .93 & .93 & .93 & .93 \\ 12.2 & .97 & .97 & .97 & .97 & .97 & .97 & .97 \\ 13.3 & 1.0 & 1.0 & 1.0 & 1.0 & 1.0 & 1.0 & 1.0 \\ 14.2 & 1.02 & 1.02 & 1.02 & 1.02 & 1.02 & 1.02 & 1.02 \\ 15.2 & 1.03 & 1.03 & 1.03 & 1.03 & 1.03 & 1.03 & 1.03 \\ 16.2 & 1.01 & 1.01 & 1.01 & 1.01 & 1.01 & 1.01 & 1.01 \\ 18.1 & .9 & .9 & .9 & .9 & .9 & .9 & .9 \\ 19.2 & .78 & .78 & .78 & .78 & .78 & .78 & .78 \\ 30.0 & 1.0 & 1.0 & 1.0 & 1.0 & 1.0 & 1.0 & 1.0\end{array}$




\begin{tabular}{|c|c|c|c|c|c|c|c|}
\hline 29. & -1. & -1 . & -1 & -1 & -1 & -1 & -1 \\
\hline 47. & & & & -1 & -1 & -1 & -1 \\
\hline & -.62 & -.62 & -.62 & -.62 & -.62 & -.62 & -.62 \\
\hline & -.78 & -.78 & -.78 & -.78 & -.78 & -.78 & -.78 \\
\hline & 0 & 0. & 0 & 0. & 0. & 0 & 0 \\
\hline & 0 . & .18 & .28 & .38 & .48 & .62 & .72 \\
\hline 80 . & .022 & .022 & .022 & .022 & .022 & .022 & .022 \\
\hline & .062 & .062 & .062 & .062 & .062 & .062 & .062 \\
\hline & .132 & 132 & 132 & .132 & 132 & 132 & .132 \\
\hline & .242 & 242 & 242 & 42 & & 42 & .242 \\
\hline & .302 & 302 & 302 & 02 & & 02 & \\
\hline & 1.042 & 1.042 & 1.042 & 1.042 & 1.042 & 1.042 & 1.042 \\
\hline & 1.652 & 1.652 & 1.652 & 1.652 & .652 & 1.652 & 1.652 \\
\hline & 1.852 & 1.852 & .852 & 1.8 & 2 & 52 & .852 \\
\hline & 2.022 & .022 & .022 & .022 & .022 & .022 & .022 \\
\hline & 2.022 & .0 & .022 & 2 & .022 & .022 & .022 \\
\hline & 1.962 & .9 & 1.962 & 52 & .962 & .962 & .962 \\
\hline & 1.842 & 1.842 & 1.842 & 1.842 & 1.842 & .842 & .842 \\
\hline & 1.662 & 1.662 & 1.662 & 1.662 & 1.662 & 1.662 & 1.662 \\
\hline & 1.392 & 1.392 & 1.392 & 1.392 & 1.392 & 1.399 & 1.392 \\
\hline & .562 & .562 & .562 & .562 & .562 & .562 & .562 \\
\hline & .332 & .332 & .332 & .332 & .332 & 32 & .332 \\
\hline & .155 & .155 & .181 & .207 & .23 & & .2 \\
\hline & .102 & .102 & .148 & .181 & .209 & .233 & .252 \\
\hline & .038 & .038 & .099 & .146 & .180 & .212 & .233 \\
\hline & .0264 & .026 & .0455 & .094 & 48 & 91 & .216 \\
\hline & .022 & .022 & & & .111 & 164 & .198 \\
\hline & .0196 & .0196 & .0232 & .0 & .0 & 135 & .1 \\
\hline & .0174 & & .0189 & .0 & .053 & .105 & .145 \\
\hline & .0154 & .01 & .01 & .01 & .03 & 077 & .122 \\
\hline & .0138 & .013 & .0138 & .01 & .0220 & 053 & .101 \\
\hline & .0122 & .0122 & .0122 & .0123 & .0141 & .035 & .082 \\
\hline & .011 & .011 & .011 & .011 & .011 & 12 & .0615 \\
\hline & .01 & .0 & .01 &. & .01 & 2 & \\
\hline & .0127 & & & & & 27 & \\
\hline & .009 & .009 & .009 & .00 & .00 &. & \\
\hline & .0085 & & & & & & \\
\hline & & &. & & & & \\
\hline & .0088 & 088 & .0088 & 088 & .0088 & 88 & 088 \\
\hline & .009 & & .009 & & .009 & . & 09 \\
\hline & .0167 & 167 & .0167 & 167 & .0167 & 167 & .0167 \\
\hline & & & .0231 & & .0231 & & .0231 \\
\hline & & & & & & & .0236 \\
\hline & & & & & & & .0368 \\
\hline & .0551 & .0 & .0551 & & .055 & & .0 \\
\hline & .06 & & .061 & & .06 & & \\
\hline & .0705 & .0705 & .0705 & .0705 & .0705 & .0705 & .0705 \\
\hline & .088 & & .088 & & .088 & & .088 \\
\hline & & & & & & & .1325 \\
\hline & .3474 & & .3474 & & .3474 & .3474 & .3474 \\
\hline & .500 & .500 & .500 & .500 & .500 & .500 & .500 \\
\hline & 1.392 & & 1.392 & & 1.3 & & 1.392 \\
\hline & 1.662 & 1.662 & 1.662 & 1.662 & 1.662 & 1.662 & 1.662 \\
\hline & 1.842 & 1.842 & 1.842 & 1.842 & 1.842 & 1.842 & 1.842 \\
\hline & 1.962 & & & & & & \\
\hline & 2.022 & & 2.022 & 2.022 & 2.022 & 2.022 & 2.022 \\
\hline & 2.022 & 2.022 & 2.022 & 2.022 & 2.022 & 2.022 & 2.022 \\
\hline
\end{tabular}




\begin{tabular}{|c|c|c|c|c|c|c|c|c|c|}
\hline 110. & 1.852 & 1.852 & 1.852 & 1.852 & 1.852 & 1.852 & 1.852 & & \\
\hline 120. & 1.652 & 1.652 & 1.652 & 1.652 & 1.652 & 1.652 & 1.652 & & \\
\hline 140. & 1.042 & 1.042 & 1.042 & 1.042 & 1.042 & 1.042 & 1.042 & & \\
\hline 160. & .302 & .302 & .302 & .302 & .302 & .302 & .302 & & \\
\hline 65. & .242 & .242 & .242 & .242 & .242 & .242 & .242 & & \\
\hline 170 . & .132 & .132 & .132 & .132 & .132 & .132 & .132 & & \\
\hline 175 & .062 & .062 & .062 & .062 & .062 & .062 & .062 & & \\
\hline 80. & .022 & .022 & .022 & .022 & .022 & .022 & .022 & & \\
\hline & .20 & .30 & .40 & .50 & .6 & .7 & .75 & .8 & .9 \\
\hline-180. & 0. & 0. & 0 & 0. & 0. & 0 . & 0 & 0 . & 0 . \\
\hline 170. & .4 & .4 & .4 & .4 & .4 & .4 & .4 & .4 & .4 \\
\hline-165 . & .3 & .3 & .3 & .3 & .3 & .3 & .3 & .3 & .3 \\
\hline-160. & .3 & .3 & .3 & .3 & .3 & .3 & .3 & .3 & .3 \\
\hline-135. & .5 & .5 & .5 & .5 & .5 & .5 & .5 & .5 & .5 \\
\hline-90 & .5 & .5 & .5 & .5 & .5 & .5 & .5 & .5 & .5 \\
\hline-30 & .174 & .184 & .196 & .214 & .235 & .25 & .264 & .277 & .298 \\
\hline-23 & .112 & .118 & .128 & .144 & .157 & .171 & .183 & .206 & .232 \\
\hline-16 & .073 & .078 & .086 & .097 & .108 & .117 & .137 & .176 & .200 \\
\hline-15. & .054 & .065 & .073 & .084 & .097 & .111 & .133 & .173 & .195 \\
\hline-14. & 0. & .027 & .054 & .068 & .086 & .103 & .127 & .167 & .189 \\
\hline-13 & 0 . & .0015 & .025 & .05 & .074 & .093 & .122 & .163 & .184 \\
\hline-12 & 0 . & 0 & .002 & .03 & .06 & .083 & .116 & .157 & .176 \\
\hline-11. & 0 . & 0 . & -.003 & .014 & .046 & .074 & .108 & .149 & .17 \\
\hline-10 & 0 . & 0 . & -.0015 & .002 & .032 & .065 & .10 & .142 & .163 \\
\hline-9 & 0 . & 0 . & 0 & -.003 & .016 & .054 & .089 & .132 & .154 \\
\hline-8 & 0 . & 0 & 0 & -.004 & .005 & .041 & .082 & .123 & .145 \\
\hline-7 . & 0 . & 0 . & 0 . & 0 & -.004 & .0275 & .072 & .1125 & .136 \\
\hline-6 & 0 . & 0 . & 0. & 0 . & -.003 & .016 & .0625 & .10 & .125 \\
\hline-4 & 0 . & 0 . & 0 . & 0 . & 0 & .005 & .04 & .076 & .102 \\
\hline-3 & 0 . & 0 & 0 . & 0 & 0 . & -.0025 & .026 & .0665 & .087 \\
\hline-2 . & 0 . & 0 . & 0 . & 0 . & 0 . & 0 & .013 & .053 & .07 \\
\hline-1 & 0 . & 0 . & 0 . & 0 . & 0 . & 0 . & .0035 & .033 & .045 \\
\hline 0. & 0 . & 0. & 0 . & 0 . & 0 . & 0 . & 0 & 0 & 0 \\
\hline 1 . & 0 . & 0 . & 0 . & 0 . & 0 . & 0 . & -.0035 & -.033 & -.045 \\
\hline 2 . & 0 . & 0 . & 0 . & 0 . & 0 . & 0 & -.013 & -.053 & -.07 \\
\hline 3 . & 0 . & 0. & 0 . & 0 . & 0 . & .0025 & -.026 & -.0665 & -.087 \\
\hline 4. & 0 . & 0 . & 0 . & 0 . & 0 . & -.005 & -.04 & -.076 & -.102 \\
\hline 6. & 0 . & 0 & 0 . & 0 . & .003 & -.016 & -.0625 & -.1 & -.125 \\
\hline 7 . & 0 . & 0 & 0 . & 0 . & .004 & -.0275 & -.072 & -.1125 & -.136 \\
\hline 8 . & 0 . & 0 . & 0 . & .004 & -.005 & -.041 & -.082 & -.123 & -.145 \\
\hline 9. & 0 . & 0 . & 0 & .003 & -.016 & -.054 & -.089 & -.132 & -.154 \\
\hline 10. & 0 . & 0 . & .0015 & -.002 & -.032 & -.065 & -.1 & -.142 & -.163 \\
\hline 11. & 0 . & 0 & .003 & -.014 & -.046 & -.074 & -.108 & -.149 & -.17 \\
\hline 12 . & 0 . & 0 & -.002 & -.03 & -.06 & -.083 & -.116 & -.157 & -.176 \\
\hline 13. & 0 . & -.0015 & -.025 & -.05 & -.074 & -.093 & -.122 & -.163 & -.184 \\
\hline 14. & 0 & -.027 & -.054 & -.068 & -.086 & -.103 & -.127 & -.167 & -.189 \\
\hline 15. & -.054 & -.065 & -.073 & -.084 & -.097 & -.111 & -.133 & -.173 & -.195 \\
\hline 16. & -.073 & -.078 & -.086 & -.097 & -.108 & -.117 & -.137 & -.176 & -.20 \\
\hline 23 . & -.112 & -.118 & -.128 & -.144 & -.157 & -.171 & -.183 & -.206 & -.232 \\
\hline 30 . & -.174 & -.184 & -.196 & -.214 & -.235 & -.250 & -.264 & -.277 & -.298 \\
\hline 90 . & -.5 & -.5 & -.5 & -.5 & -.5 & -.5 & -.5 & -.5 & -.5 \\
\hline 135. & -.5 & -.5 & -.5 & -.5 & -.5 & -.5 & -.5 & -.5 & -.5 \\
\hline 160. & -.3 & -.3 & -.3 & -.3 & -.3 & -.3 & -.3 & -.3 & -.3 \\
\hline & -.3 & -.3 & -.3 & -.3 & -.3 & -.3 & -.3 & -.3 & -.3 \\
\hline & -.4 & -.4 & -.4 & -.4 & -.4 & -.4 & -.4 & -.4 & -.4 \\
\hline 180. & 0 & 0 & 0 & 0 . & 0. & 0. & 0 & 0 & 0 \\
\hline
\end{tabular}


NACA0012 for Puma

from Bousman, US Army, October 1988

corrected cd typo at alpha=-10; October 1988

\section{RCAS Input File}

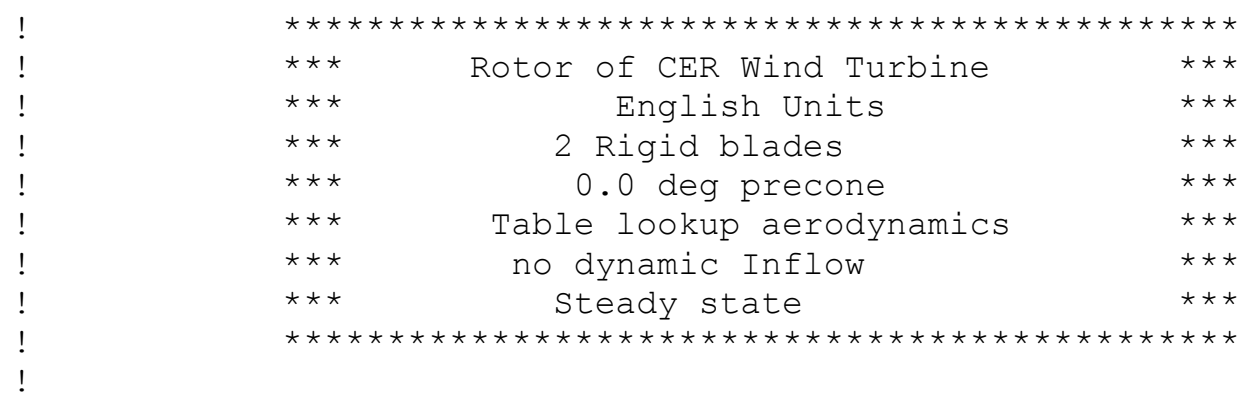

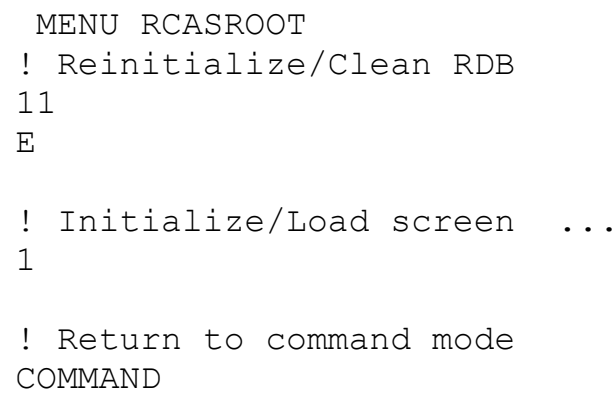




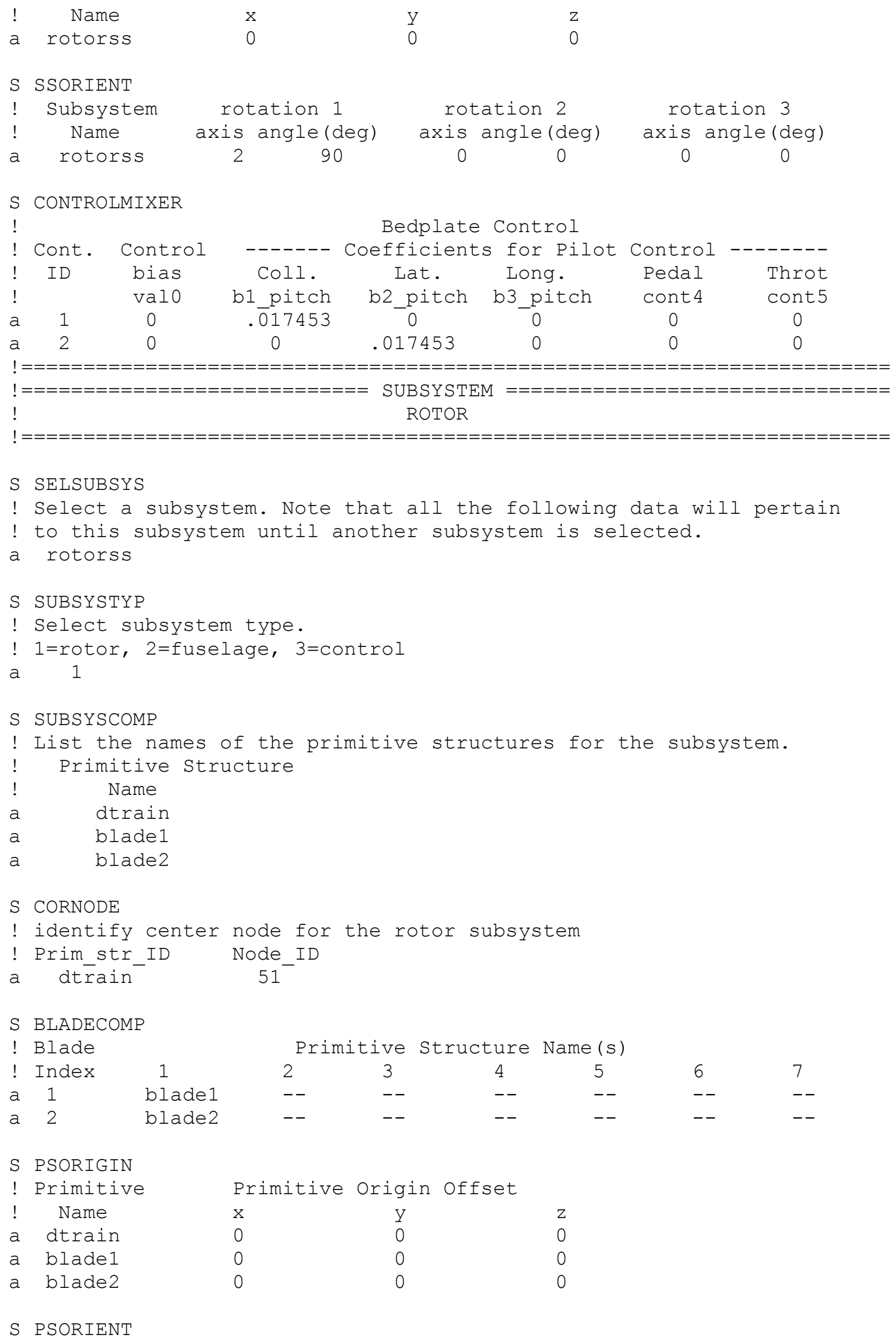




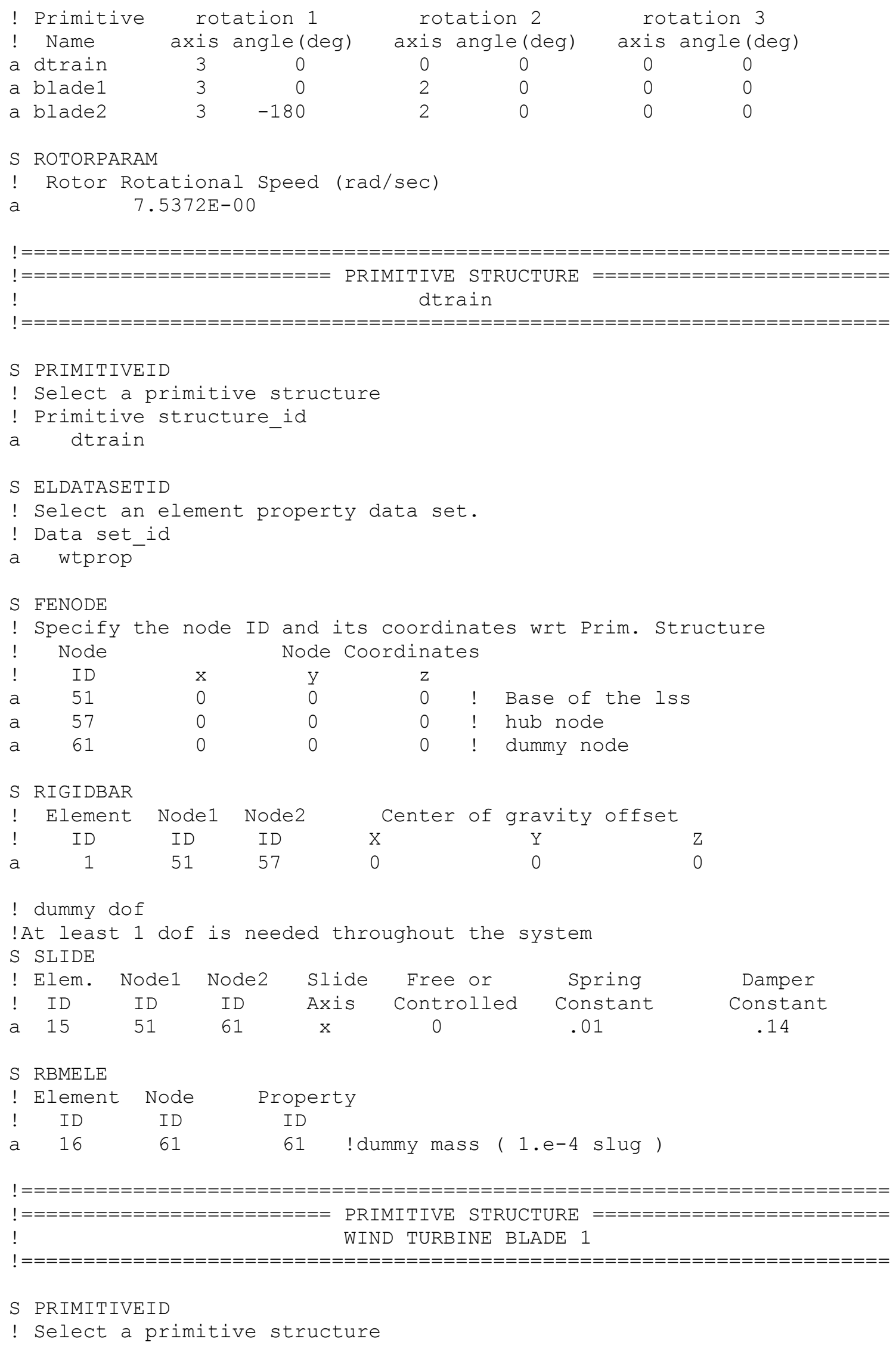




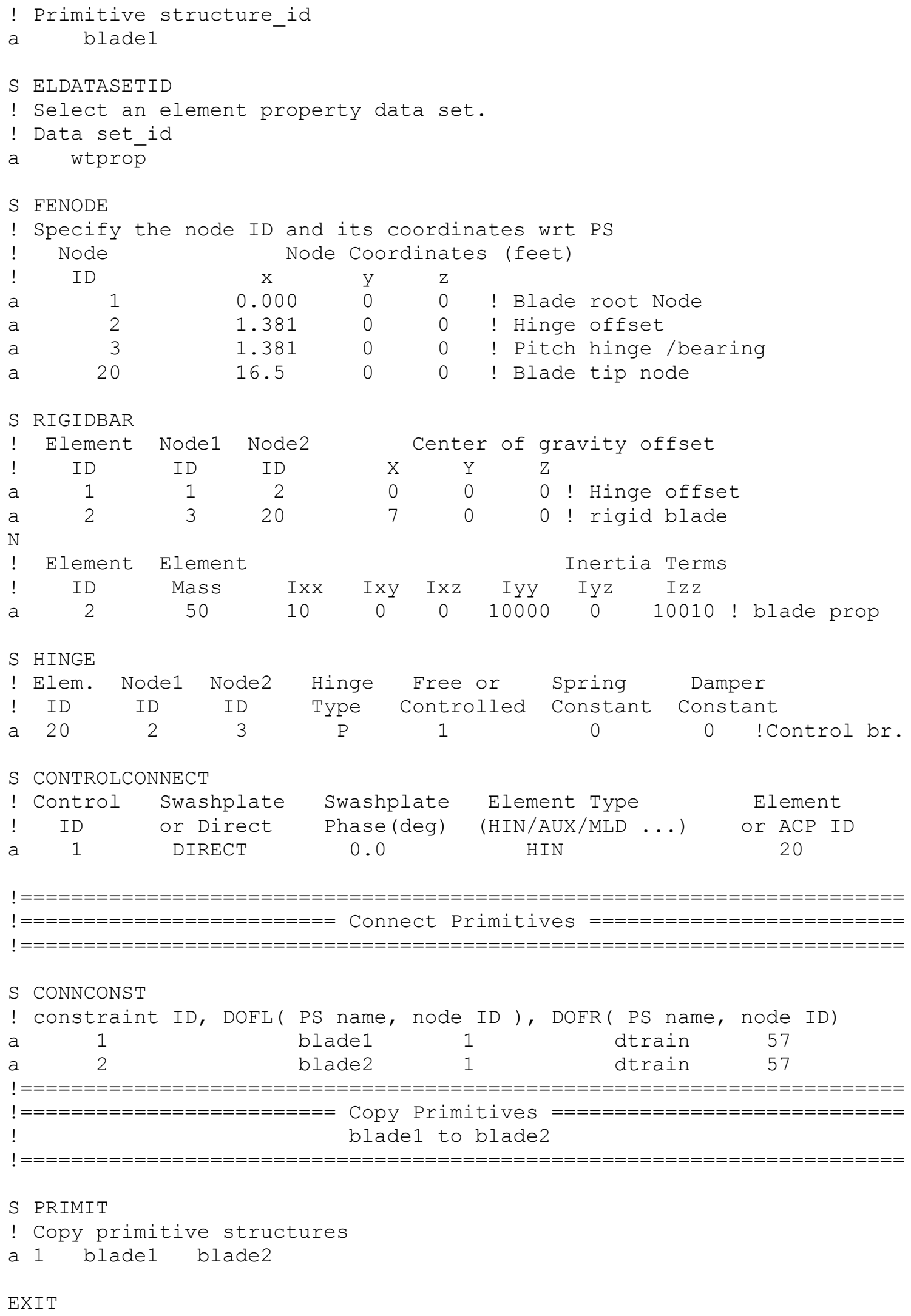


copyprimstruct

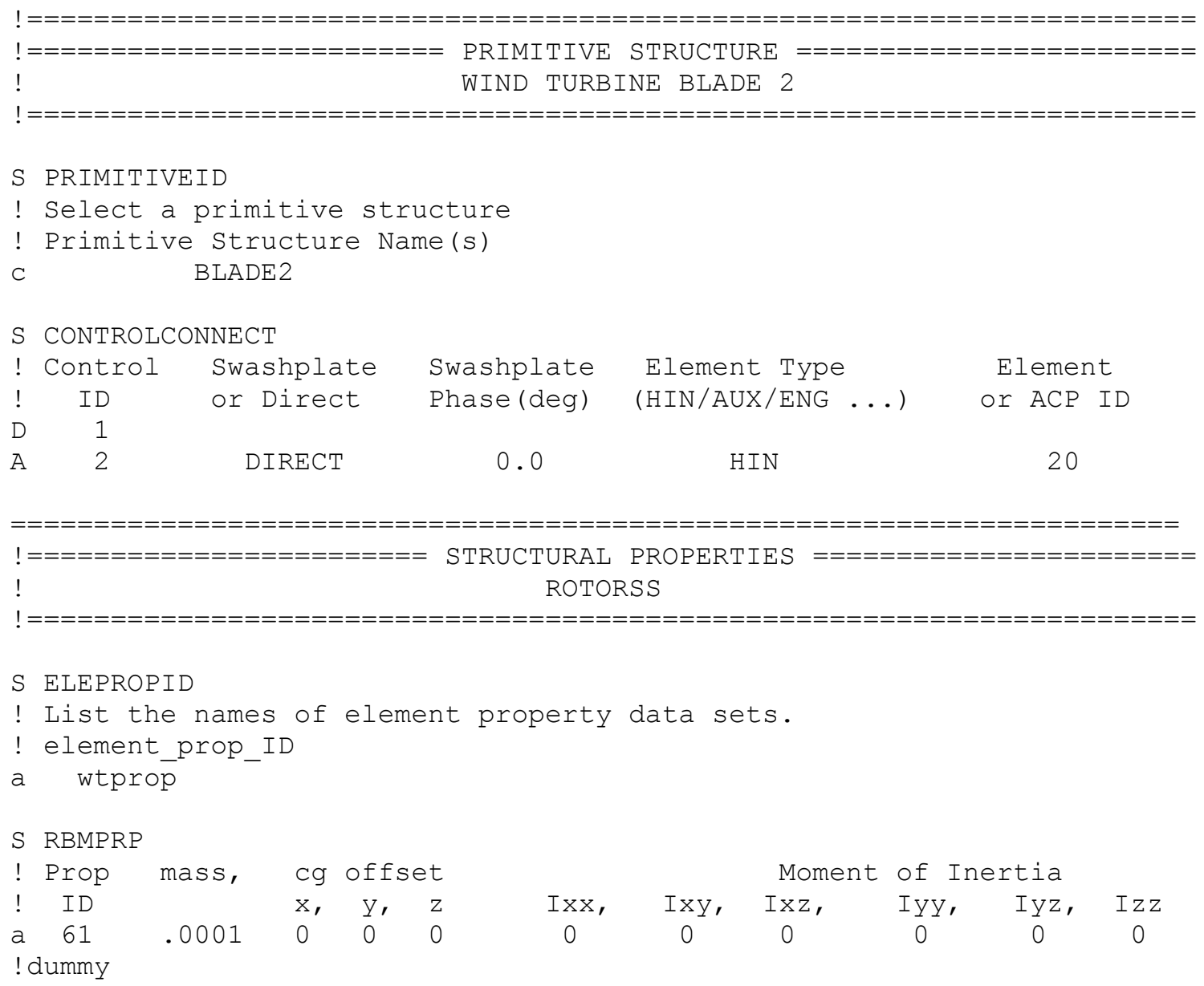

S AEROMODCOMP 


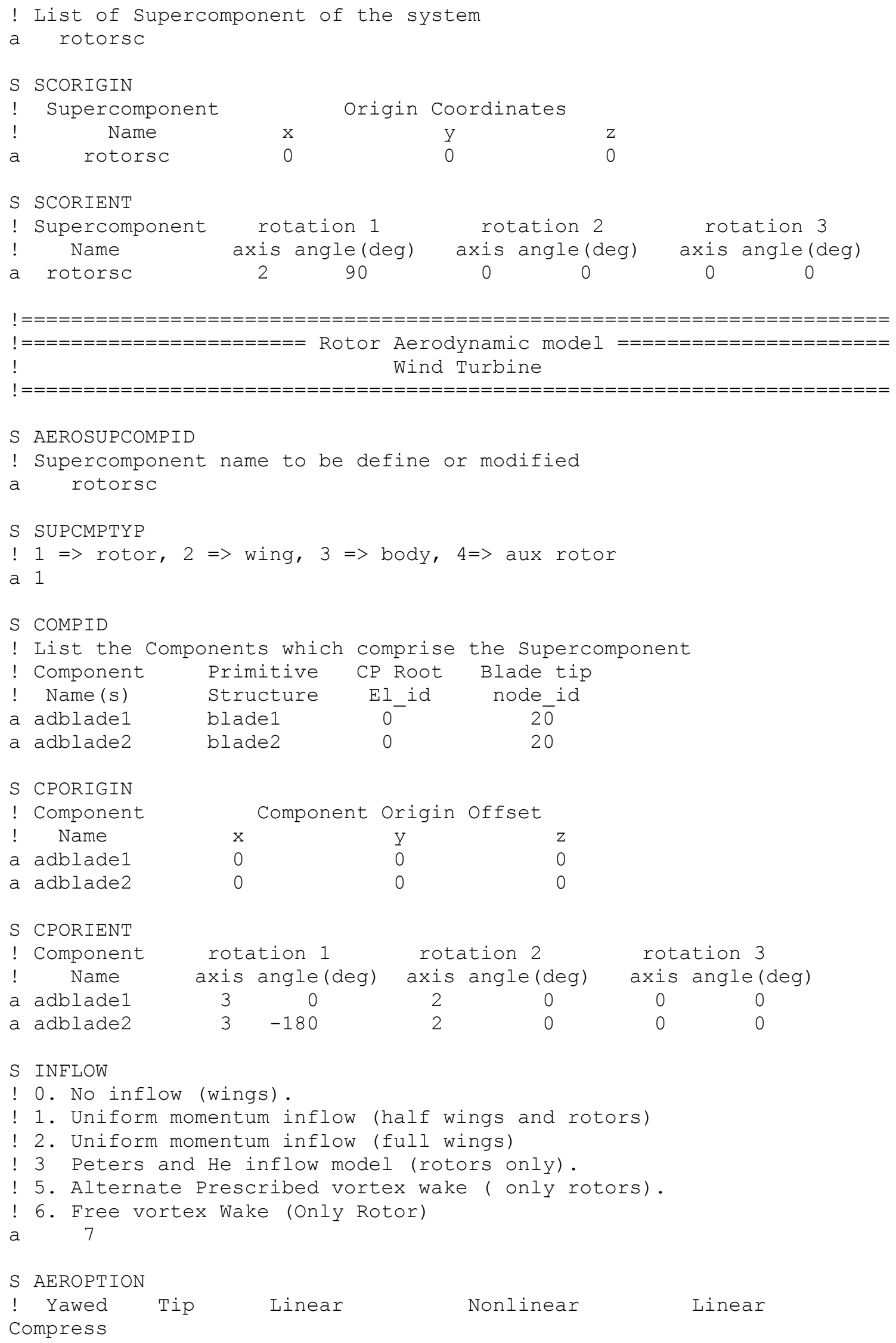




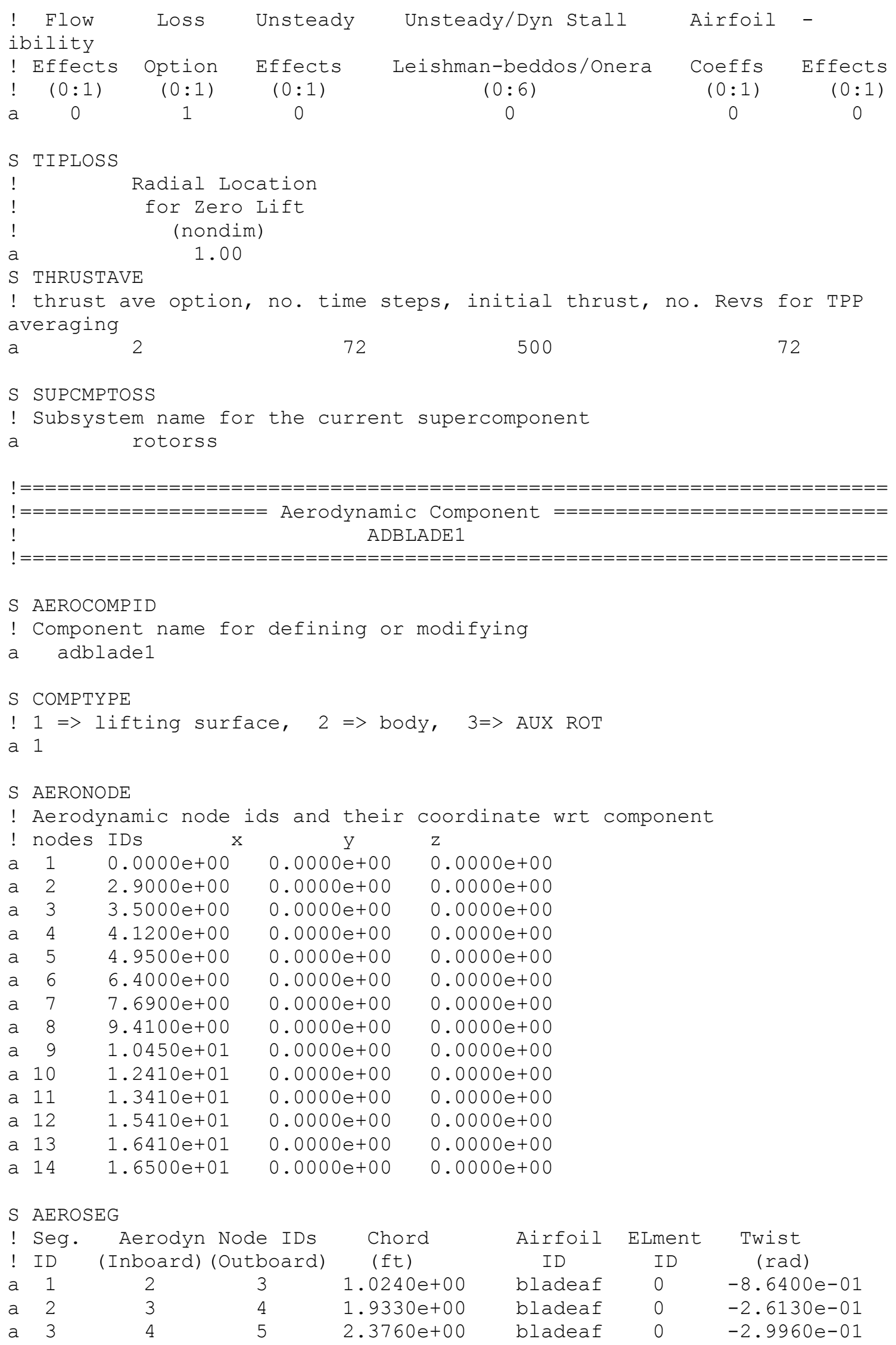




\begin{tabular}{|c|c|c|c|c|c|c|}
\hline 4 & 5 & 6 & $2.2590 e+00$ & bladeaf & 0 & $-1.9430 e-01$ \\
\hline 5 & 6 & 7 & $2.1210 e+00$ & bladeaf & 0 & $-1.1080 e-01$ \\
\hline 6 & 7 & 8 & $1.9700 \mathrm{e}+00$ & bladeaf & 0 & $-5.9300 e-02$ \\
\hline 7 & 8 & 9 & $1.8310 e+00$ & bladeaf & 0 & $-2.7900 e-02$ \\
\hline 8 & 9 & 10 & $1.6800 e+00$ & bladeaf & 0 & $-1.0100 e-02$ \\
\hline 9 & 10 & 11 & $1.5310 \mathrm{e}+00$ & bladeaf & 0 & $4.3600 e-03$ \\
\hline a 10 & 11 & 12 & $1.3780 e+00$ & bladeaf & 0 & $1.5970 e-02$ \\
\hline a 11 & 12 & 13 & $1.2260 e+00$ & bladeaf & 0 & $2.7310 e-02$ \\
\hline a 12 & 13 & 14 & $1.1710 \mathrm{e}+00$ & bladeaf & 0 & $3.1240 e-02$ \\
\hline
\end{tabular}

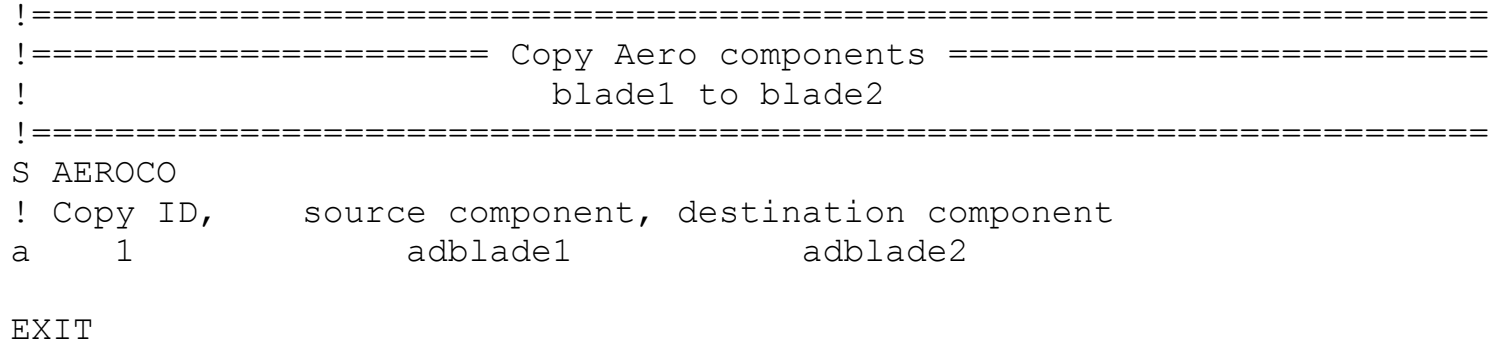




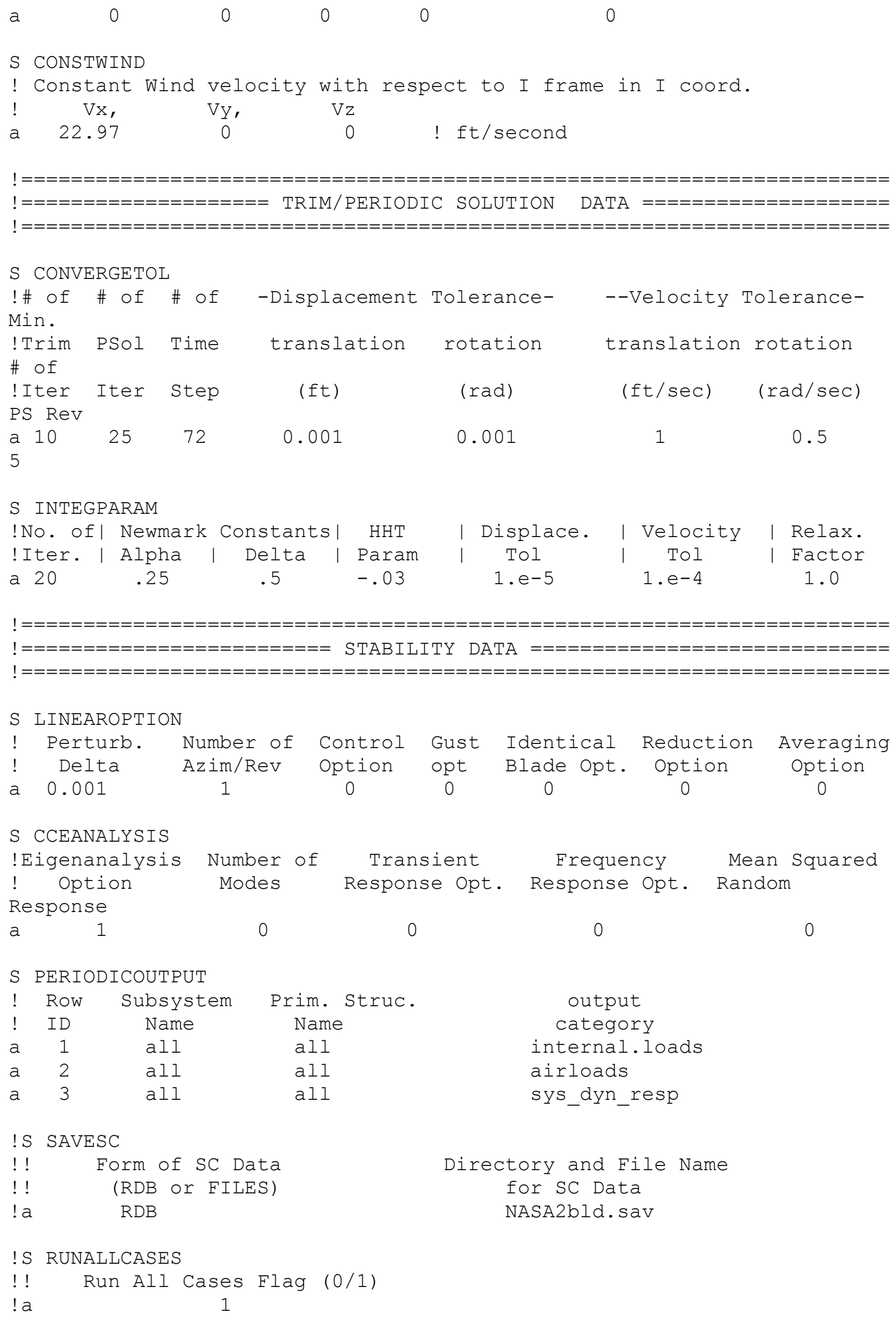




\section{WT_Perf Input File}

WT_Perf v2.2 input file:

----- Job Title (one line)

WT_Perf test input file. CER/NASA Phase 4 turbine. Delft wind tunnel data.

$\begin{array}{lc}\text { TRUE } & \text { ECHO_INP: Echo input parameters to echo.out? } \\ \text { FALSE } & \text { DIMEN: } \quad \text { Turbine parameters are dimensional? } \\ \text { FALSE } & \text { METRIC: Turbine parameters are metric (MKS vs FPS]? }\end{array}$

\begin{tabular}{|c|c|}
\hline & t Configuration -- \\
\hline TRUE & TABDELIM: Make output tab-delimited (fixed-width otherwise). \\
\hline TRUE & WRITE_BE: Write out blade element data to bladelem.dat? \\
\hline 3 & PAR_RŌW: Row parameter (1-rpm, 2-pitch, 3-tsr/speed). \\
\hline 2 & PAR_COL: Column parameter (1-rpm, 2-pitch, 3-tsr/speed). \\
\hline 1 & PAR_SHT: Sheet parameter (1-rpm, 2-pitch, 3-tsr/speed). \\
\hline TRUE & OUT_PWR: Request output of rotor power? \\
\hline TRUE & OUT_CP: Request output of Cp? \\
\hline TRUE & OUT_TRQ: Request output of shaft torque? \\
\hline FALSE & OUT_FLP: Request output of flap bending moment? \\
\hline TRUE & OUT_THR: Request output of rotor thrust? \\
\hline & 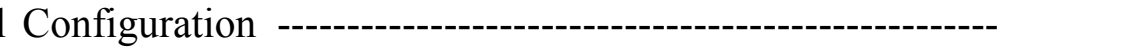 \\
\hline 20 & N_SEG: Number of blade segments (entire rotor radius). \\
\hline 5 & SEG_FRST: First segment used in the analysis. \\
\hline 20 & SEG_LAST: Last segment used in the analysis. \\
\hline 1 & N_SECT: Number of circumferential sectors. \\
\hline 50 & MAX_ITER: Max number of iterations for induction factor. \\
\hline ---- & 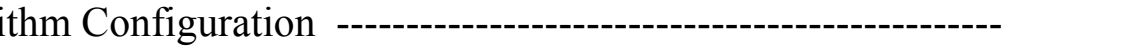 \\
\hline TRUE & VITERNA: Use Viterna post stall instead of flat plate? \\
\hline TRUE & TIP_LOSS: Use the Prandtl tip loss model? \\
\hline TRUE & HUB_LOSS: Use the Prandtl hub loss model? \\
\hline TRUE & SWIRL: Include swirl effects? \\
\hline TRUE & ADV_BRK: Use the advanced brake-state model? \\
\hline FALSE & ADD_3D: Add ECN's 3D effects to $\mathrm{Cl}$ and $\mathrm{Cd}$ ? \\
\hline
\end{tabular}


72, 72, 0 OMG_ST, OMG_END, OMG_DEL: First, last, delta rotor speed.

FALSE INP TSR: Input speeds as TSRs?

5, 20,1 SPD_ST, SPD_END, SPD_DEL: First, last, delta speeds.

mps $\quad$ UNITS: $\quad$ Wind speed units (mps, fps, $\mathrm{mph}$ ).

\begin{tabular}{|c|c|c|c|}
\hline \multicolumn{4}{|c|}{ 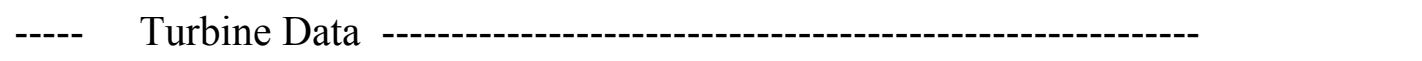 } \\
\hline 2 & & \multicolumn{2}{|c|}{ NUM BL: $\quad$ Number of blades. } \\
\hline 16.5 & & \multicolumn{2}{|c|}{ ROT_RAD: $\quad$ Rotor radius [length]. } \\
\hline-1.63 & 0.0 & \multicolumn{2}{|c|}{ TIPTWIST: $\quad$ Angle between tip chordline and rotor plane [deg]. } \\
\hline 0.04 & 0.21 & $0.0 \quad \mathrm{CHC}$ & \multirow{2}{*}{$\begin{array}{l}\text { CHORD, THICK, TWIST: N_SEG lines of Chord [length or } \\
\text { div by radius], Thickness [length }\end{array}$} \\
\hline 0.04 & 0.21 & $\operatorname{div} 1$ & \\
\hline 0.04 & 0.21 & or $\mathrm{d}$ & or div by chord], and Twist [deg]. \\
\hline 0.04 & 0.21 & \multicolumn{2}{|r|}{ 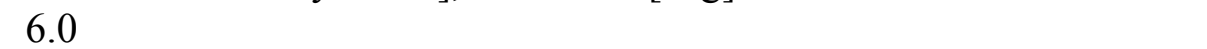 } \\
\hline \multicolumn{4}{|c|}{0.10657} \\
\hline 0.144 & & \multicolumn{2}{|l|}{17.0} \\
\hline 0.1389 & & \multicolumn{2}{|l|}{$0.21 \quad 12.2$} \\
\hline 0.1338 & & \multicolumn{2}{|l|}{0.218 .7} \\
\hline 0.1288 & & \multicolumn{2}{|l|}{$0.21 \quad 6.18$} \\
\hline 0.1237 & & \multicolumn{2}{|l|}{0.214 .36} \\
\hline 0.1187 & & \multicolumn{2}{|l|}{$0.21 \quad 3.03$} \\
\hline 0.1136 & & \multicolumn{2}{|l|}{0.212 .04} \\
\hline 0.1086 & & \multicolumn{2}{|l|}{$0.21 \quad 1.27$} \\
\hline 0.1035 & & \multicolumn{2}{|l|}{$0.21 \quad 0.66$} \\
\hline 0.0985 & & \multicolumn{2}{|l|}{$0.21 \quad 0.16$} \\
\hline 0.0934 & & \multicolumn{2}{|l|}{$0.21-0.26$} \\
\hline 0.0883 & & \multicolumn{2}{|l|}{$0.21-0.62$} \\
\hline 0.0833 & & \multicolumn{2}{|l|}{$0.21-0.94$} \\
\hline 0.0782 & & \multicolumn{2}{|l|}{$0.21-1.26$} \\
\hline 0.0732 & & \multicolumn{2}{|l|}{$0.21-1.63$} \\
\hline 0.144 & & \multirow{2}{*}{\multicolumn{2}{|c|}{$\begin{array}{l}\text { HUB_RAD: } \quad \text { Hub radius [length or div by radius]. } \\
\text { PRECONE: }\end{array}$}} \\
\hline 0.0 & & & \\
\hline 0.0 & & \multirow{2}{*}{\multicolumn{2}{|c|}{$\begin{array}{ll}\text { TILT: } & \text { Shaft tilt (can be used as yaw if shear is zero). } \\
\text { HUB HT: } & \text { Hub height [length or div by radius]. }\end{array}$}} \\
\hline 3.3333 & & & \\
\hline ----- & Aero & dynamic Data & ---------------------------------------------------------- \\
\hline 0.0023 & & RHO: & Air density [mass/volume]. \\
\hline 0.0 & & SHR_EXP: & Wind shear exponent $(1 / 7$ law $=0.143)$ \\
\hline FALS & & SEP & _TABL: $\quad$ Are $\mathrm{Cd}$ and $\mathrm{Cl}$ tables separate? \\
\hline 1,24, & & $\mathrm{SEG}, \mathrm{NCL}, \overline{\mathrm{T}}$ & NCD: Segment\#, \#CLs, \#CDs (Reuse segment NCD if \\
\hline $\mathrm{NCL}=$ & & & \\
\hline 15.24 & & ALF_STAL: & : $\quad$ Stall angle of attack (deg). Peak of CL curve? \\
\hline 1 & & I_SHFT: & For 3D, index of $\mathrm{Cl}$ curve where we depart from linear. \\
\hline-1.04 & 0.019 & $0.0095 \mathrm{CL}$ & L, CL, CD: NCL lines of Alpha (deg), Cl, and Cd. \\
\hline-0.01 & 0.139 & 0.0094 & \\
\hline 1.02 & 0.258 & 0.0096 & \\
\hline 2.05 & 0.378 & 0.0099 & \\
\hline
\end{tabular}




$\begin{array}{lcl}3.07 & 0.497 & 0.01 \\ 4.1 & 0.617 & 0.01 \\ 5.13 & 0.736 & 0.0097 \\ 6.16 & 0.851 & 0.0095 \\ 7.18 & 0.913 & 0.0127 \\ 8.2 & 0.952 & 0.0169 \\ 9.21 & 0.973 & 0.0247 \\ 10.2 & 0.952 & 0.0375 \\ 11.21 & 0.947 & 0.0725 \\ 12.23 & 1.007 & 0.0636 \\ 13.22 & 1.031 & 0.0703 \\ 14.23 & 1.055 & 0.0828 \\ 15.23 & 1.062 & 0.1081 \\ 16.22 & 1.043 & 0.1425 \\ 20.00 & 0.700 & 0.3 \\ 30 & 1 & 0.580 \\ 45 & 1 & 1 \\ 90 & 0.1 & 1.6\end{array}$

2, 0, 1 SEG, NCL, NCD: Segment\#, \#CLs, \#CDs (Reuse segment NCD if NCL=0). 3, 0, 1 SEG, NCL, NCD: Segment\#, \#CLs, \#CDs (Reuse segment NCD if NCL=0). 4, 0, 1 SEG, NCL, NCD: Segment\#, \#CLs, \#CDs (Reuse segment NCD if NCL=0). 5, 0, 1 SEG, NCL, NCD: Segment\#, \#CLs, \#CDs (Reuse segment NCD if NCL=0). 7, 0, 1 SEG, NCL, NCD: Segment\#, \#CLs, \#CDs (Reuse segment NCD if NCL=0). 8, 0, 1 SEG, NCL, NCD: Segment\#, \#CLs, \#CDs (Reuse segment NCD if NCL=0). 9, 0, 1 SEG, NCL, NCD: Segment\#, \#CLs, \#CDs (Reuse segment NCD if NCL=0). 10, 0, 1 SEG, NCL, NCD: Segment\#, \#CLs, \#CDs (Reuse segment NCD if NCL=0). 11, 0, 1 SEG, NCL, NCD: Segment\#, \#CLs, \#CDs (Reuse segment NCD if NCL=0). 12, 0, 1 SEG, NCL, NCD: Segment\#, \#CLs, \#CDs (Reuse segment NCD if NCL=0). 13, 0, 1 SEG, NCL, NCD: Segment\#, \#CLs, \#CDs (Reuse segment NCD if NCL=0). 14, 0, 1 SEG, NCL, NCD: Segment\#, \#CLs, \#CDs (Reuse segment NCD if NCL=0). 15, 0, 1 SEG, NCL, NCD: Segment\#, \#CLs, \#CDs (Reuse segment NCD if NCL=0). 16, 0, 1 SEG, NCL, NCD: Segment\#, \#CLs, \#CDs (Reuse segment NCD if NCL=0). 17, 0, 1 SEG, NCL, NCD: Segment\#, \#CLs, \#CDs (Reuse segment NCD if NCL=0). 18, 0, 1 SEG, NCL, NCD: Segment\#, \#CLs, \#CDs (Reuse segment NCD if NCL=0). 19, 0, 1 SEG, NCL, NCD: Segment\#, \#CLs, \#CDs (Reuse segment NCD if NCL=0). 20, 0, 1 SEG, NCL, NCD: Segment\#, \#CLs, \#CDs (Reuse segment NCD if NCL=0).

\section{AeroDyn/YawDyn Input File}

Combined Experiment Baseline for YawDyn version 12.1

ENGLISH Units for input and output [SI or ENGlish]

STEADY Dynamic stall model [BEDDOES or STEADY]

NO_CM Aerodynamic pitching moment model [USE_CM or NO_CM]

EQUIILInflow model [DYNIN or EQUIL]

SWIRL Induction factor model [NONE or WAKE or SWIRL]

5.0000E-03 Convergence tolerance for induction factor 
PRAND Tip-loss model (EQUIL only) [PRANdtl, GTECH, or NONE]

PRAND Hub-loss model (EQUIL only) [PRANdtl or NONE]

"STEADYtt.wnd" Hub-height steady wind file

55.0 Wind reference (hub) height.

0.0 Tower shadow centerline velocity deficit.

1.0 Tower shadow half width.

0.0 Tower shadow reference point.

2.3870E-03 Air density.

$1.625 \mathrm{e}-4 \quad$ KinVisc - Kinematic air viscosity

1.0000E-03 Time interval for aerodynamic calculations.

1 Number of airfoil files used. Files listed below:

"S809d_Cln.dat"

$20 \quad$ Number of blade elements per blade

RELM Twist DR Chord File IDElem Data RELM and Twist ignored by

ADAMS (but placeholders must be present)

$\begin{array}{lcrlll}0.4125 & 0 & 0.825 & 0.66 & 1 & \\ 1.2375 & 0 & 0.825 & 0.66 & 1 & \\ 2.0625 & 0 & 0.825 & 0.66 & 1 & \\ 2.8875 & 6 & 0.825 & 0.66 & 1 & \\ 3.7125 & 13.4 & 0.825 & 1.7584 & 1 & \\ 4.5375 & 17 . & 0.825 & 2.376 & 1 & \text { PRINT } \\ 5.3625 & 12.2 & 0.825 & 2.2925 & 1 & \text { PRINT } \\ 6.1875 & 8.7 & 0.825 & 2.2092 & 1 & \text { PRINT } \\ 7.0125 & 6.18 & 0.825 & 2.1257 & 1 & \text { PRINT } \\ 7.8375 & 4.36 & 0.825 & 2.0424 & 1 & \text { PRINT } \\ 8.6625 & 3.03 & 0.825 & 1.9589 & 1 & \text { PRINT } \\ 9.4875 & 2.04 & 0.825 & 1.8756 & 1 & \text { PRINT } \\ 10.3125 & 1.27 & 0.825 & 1.7921 & 1 & \text { PRINT } \\ 11.1375 & 0.66 & 0.825 & 1.7087 & 1 & \text { PRINT } \\ 11.9625 & 0.16 & 0.825 & 1.6253 & 1 & \text { PRINT } \\ 12.7875 & -0.26 & 0.825 & 1.5419 & 1 & \text { PRINT } \\ 13.6125 & -0.62 & 0.825 & 1.4584 & 1 & \text { PRINT } \\ 14.4375 & -0.94 & 0.825 & 1.3751 & 1 & \text { PRINT } \\ 15.2625 & -1.26 & 0.825 & 1.2916 & 1 & \text { PRINT } \\ 16.0875 & -1.63 & 0.825 & 1.2083 & 1 & \text { PRINT }\end{array}$

Combined Experiment Baseline in ENGLISH units for AeroDyn version 12.1

6.0 Time duration of the simulation $(\mathrm{sec})$

60.0 Number of azimuth sectors used for integration

2 Decimation factor for output printing

1.0000E-02 TOLER, Trim solution tolerance (deg)

2 Number of blades

4.8 4.8 4.8 Initial pitch angles (deg)

4.0 Rotor hub sling (distance from yaw axis to hub; positive downwind) (ft)

0.0 Shaft tilt angle (deg) 
0.0 Rotor precone angle (deg)

72.0 RPM, rotor speed in revolutions per minute

0.0 PsiInit, Initial rotor position (zero for Blade 1 down) (deg)

FIXEDYaw Model: FREE or FIXED yaw system

$0.0 \quad$ Initial yaw angle (deg)

0.0 Initial yaw rate $(\mathrm{deg} / \mathrm{sec})$

1000.0 Mass moment of inertia about yaw axis (slug- $\left.\mathrm{ft}^{\wedge} 2\right)$

0.0 YawStiff, stiffness of yaw spring (lb-ft/rad)

0.0 YawDamp, yaw damping coefficient (lb-ft-sec)

0.0 YawFriction, constant friction moment at yaw axis (lb-ft)

RIGID Hub model: HINGE, TEETER or RIGID

$0.00 .00 .0 \quad$ Initial flap angles $(\mathrm{deg})$

$0.00 .00 .0 \quad$ Initial flap rates $(\mathrm{deg} / \mathrm{sec})$

0.0 RHinge, radius of rotor hub (ft)

5.44 RBar, distance from hinge to blade c.g. (ft)

3.34 Mass of one blade (slug)

178.0 Mass moment of inertia of blade about hinge axis (slug- $\mathrm{ft}^{\wedge} 2$ )

$1.5500 \mathrm{E}+05$ Torsional stiffness of blade root spring (lbf- $\mathrm{ft} / \mathrm{rad}$ )

0.0 Teeter sling distance of teeter axis upwind of rotor apex (m)

$0.0 \quad$ Free teeter angle (deg)

0.0 Teeter stiffness, first or linear coeff. (lbf-ft/rad)

0.0 Teeter stiffness, coeff. of deflection (lbf-ft/ $\left.\mathrm{rad}^{\wedge} 2\right)$

0.0 Teeter damping coefficient (lbf-ft-sec)

$1,2,5$

$1=$ Horizontal wind speed at hub center, len/s. [HHWSpeed]

$2=$ Horizontal wind direction at hub center, deg. [HHWDir]

$3=$ Nacelle yaw angle, deg. [YawAng]

$4=$ Nacelle yaw rate, $\mathrm{deg} / \mathrm{sec}$. [YawRate]

5 = Blade azimuth angle ( 0 when blade 1 down $)$ deg. [AzimAngB1D]

$6=$ Blade azimuth angle ( 0 when blade $1 \mathrm{up})$, deg. [AzimAngB1U]

$7=$ Teeter angle, deg. [TeeterAng]

$8=$ Teeter rate, $\mathrm{deg} / \mathrm{sec}$. [TeeterRate]

10 = Blade 1 flap angle, deg. [FlapAng1]

11 = Blade 1 flap rate, $\mathrm{deg} / \mathrm{sec}$. [FlapRate1]

12 = Blade 2 flap angle, deg. [FlapAng2]

13 = Blade 2 flap rate, $\mathrm{deg} / \mathrm{sec}$. [FlapRate2]

14 = Blade 3 flap angle, deg. [FlapAng3]

15 = Blade 3 flap rate, deg/sec. [FlapRate3]

$16=$ Rotor power, $\mathrm{kW}$. [Power]

$17=$ Rotor torque, force*len. [Torque]

$20=$ Nacelle yaw moment, force*len. [YawMom]

$21=$ Nacelle yaw moment, kiloforce*len. [YawMomK]

$22=$ Hub moment, force*len. [HubMom]

$23=$ Hub moment, kiloforce*len. [HubMomK]

$24=$ Rotor thrust, force. [Thrust]

$25=$ Rotor thrust, kiloforce. [ThrustK] 
$26=$ Lateral hub force, force. [HForceY]

$27=$ Lateral hub force, kiloforce. [HForceYK]

$28=$ Vertical hub force, force. [HForceZ]

$29=$ Vertical hub force, kiloforce. [HForceZK]

$30=$ Out-of-plane bending moment for blade 1, force*len. [OutPIMom1]

31 = Out-of-plane bending moment for blade 2, force*len. [OutPIMom2]

$32=$ Out-of-plane bending moment for blade 3, force*len. [OutPIMom3]

33 = In-plane bending moment for blade 1, force*len. [InPIMom1]

$34=$ In-plane bending moment for blade 2, force*len. [InPIMom2]

$35=$ In-plane bending moment for blade 3, force*len. [InPIMom3]

$36=$ Pitching moment for blade 1 , force*len. [PitchMom1]

$37=$ Pitching moment for blade 2, force*len. [PitchMom2]

$38=$ Pitching moment for blade 3 , force*len. [PitchMom3]

$40=$ Out-of-plane bending moment for blade 1, kiloforce*len. [OutPIMom1K]

41 = Out-of-plane bending moment for blade 2, kiloforce*len. [OutPIMom2K]

42 = Out-of-plane bending moment for blade 3, kiloforce*len. [OutPIMom3K]

$43=$ In-plane bending moment for blade 1, kiloforce*len. [InPIMom1K]

$44=$ In-plane bending moment for blade 2, kiloforce*len. [InPIMom2K]

$45=$ In-plane bending moment for blade 3, kiloforce*len. [InPIMom3K]

$46=$ Pitching moment for blade 1, kiloforce*len. [PitchMom1K]

$47=$ Pitching moment for blade 2, kiloforce*len. [PitchMom2K]

$48=$ Pitching moment for blade 3, kiloforce*len. [PitchMom3K]

S809 Airfoil, Delft data at $\mathrm{Re}=1.0$ Million, Clean no roughness

NREL/TP-442-7817 Appendix B, Viterna used aspect ratio=11

1 Number of airfoil tables in this file

.00 Table ID parameter

15.30 Stall angle (deg)

.00 No longer used, enter zero

.00 No longer used, enter zero

.00 No longer used, enter zero

-.38 Zero lift angle of attack (deg)

7.12499 Cn slope for zero lift (dimensionless)

$1.9408 \mathrm{Cn}$ at stall value for positive angle of attack

$-.8000 \quad \mathrm{Cn}$ at stall value for negative angle of attack

2.0000 Angle of attack for minimum CD (deg)

.0116 Minimum CD value

$\begin{array}{llll}-180.00 & .000 & .1748 & .0000\end{array}$

$\begin{array}{llll}-170.00 & .230 & .2116 & .4000\end{array}$

$\begin{array}{llll}-160.00 & .460 & .3172 & .1018\end{array}$

$\begin{array}{llll}-150.00 & .494 & .4784 & .1333\end{array}$

$\begin{array}{llll}-140.00 & .510 & .6743 & .1727\end{array}$

$\begin{array}{llll}-130.00 & .486 & .8799 & .2132\end{array}$

$\begin{array}{llll}-120.00 & .415 & 1.0684 & .2498\end{array}$

$\begin{array}{llll}-110.00 & .302 & 1.2148 & .2779\end{array}$

$\begin{array}{llll}-100.00 & .159 & 1.2989 & .2933\end{array}$ 


\begin{tabular}{|c|c|c|c|c|}
\hline-90.00 & .000 & 1.308 & & .2936 \\
\hline-80.00 & -.159 & 1.298 & & .2933 \\
\hline-70.00 & -.302 & 1.214 & & .2779 \\
\hline-60.00 & -.415 & 1.068 & & .2498 \\
\hline-50.00 & -.486 & .879 & 2132. & \\
\hline-40.00 & -.510 & .674 & $43 \quad .1727$ & \\
\hline-30.00 & -.494 & .478 & 84.1333 & \\
\hline-20.10 & -.560 & $.302^{\prime}$ & $27 \quad .0612$ & \\
\hline-18.10 & -.670 & .306 & 0904. 69 & \\
\hline-16.10 & -.790 & .192 & $28 \quad .0293$ & \\
\hline-14.20 & -.840 & .089 & $98-.0090$ & \\
\hline-12.20 & -.700 & .055 & $53-.0045$ & \\
\hline-10.10 & -.630 & .039 & $90-.0044$ & \\
\hline-8.20 & -.560 & .023 & $33-.0051$ & \\
\hline-6.10 & -.640 & .013 & $\begin{array}{ll}31 \quad .0018\end{array}$ & \\
\hline-4.10 & -.420 & .013 & $34-.0216$ & \\
\hline-2.10 & -.160 & .009 & $90 \quad-.0282$ & \\
\hline-1.04 & 0.0190 & .0095 & -.0346 & \\
\hline-0.01 & 0.1390 & .0094 & -.0405 & \\
\hline 1.02 & 0.2580 & 0096 & -.0455 & \\
\hline 2.05 & 0.3780 & 0099 & -.0507 & \\
\hline 3.07 & 0.4970 & $01-$ & -.0404 & \\
\hline 4.10 & 0.6170 & $01-$ & -.0321 & \\
\hline 5.13 & 0.7360 & 0097 & -.0281 & \\
\hline 6.16 & 0.8510 & 0095 & -.0284 & \\
\hline 7.18 & 0.9130 & 0127 & -.0322 & \\
\hline 8.2 & 0.9520 . & 0169 & -.0361 & \\
\hline 9.21 & 0.9730 & 0247 & -.0363 & \\
\hline 10.2 & 0.9520 & 0375 & -.0393 & \\
\hline 11.21 & 0.947 & .0725 & -.0398 & \\
\hline 12.23 & 1.007 & .0636 & -.0983 & \\
\hline 13.22 & 1.031 & .0703 & -.1242 & \\
\hline 14.23 & 1.055 & .0828 & -.1155 & \\
\hline 15.23 & 1.062 & .1081 & -.2459 & \\
\hline 16.22 & 1.043 & .1425 & -.2813 & \\
\hline 20.00 & 0.700 & .30 & -.3134 & \\
\hline 30.00 & .705 & .478 & $34 \quad-.2459$ & \\
\hline 40.00 & .729 & .674 & $43-.2813$ & \\
\hline 50.00 & .694 & .879 & $99-.3134$ & \\
\hline 60.00 & .593 & 1.068 & & -.3388 \\
\hline 70.00 & .432 & 1.214 & & -.3557 \\
\hline 80.00 & .227 & 1.298 & & -.3630 \\
\hline 90.00 & .000 & 1.308 & & -.3604 \\
\hline 100.00 & -.159 & 1.298 & & -.3600 \\
\hline 110.00 & -.302 & 1.214 & & -.3446 \\
\hline 120.00 & -.415 & 1.068 & 84 & -.3166 \\
\hline
\end{tabular}




\begin{tabular}{|c|c|c|}
\hline 130.00 & -.486 & ا.2800 \\
\hline 140.00 & -.510 & $.6743-.23$ \\
\hline 150.00 & -.494 & $.4784-$ \\
\hline 160.00 & -.460 & $.3172-.16$ \\
\hline 170.00 & -.230 & $.2116-.5$ \\
\hline 180.00 & .000 & .00 $1748 \quad$. \\
\hline
\end{tabular}

! Steady wind file for $30 \mathrm{fps}$ ( or $\mathrm{m} / \mathrm{s}$ ) wind speed for AeroDyn

! Time Wind Wind Vert. Horiz. Vert. LinV Gust

! Speed Dir Speed Shear Shear Shear Speed

$\begin{array}{llllllll}0.0 & 22.97 & 0.0 & 0 & 0.0 & 0.0 & 0 & 0\end{array}$

LSWT Input File

\begin{tabular}{|c|c|c|c|c|c|c|}
\hline 2 BLADED & NREL/NASA & COMBINED & EXPERIMENT & ROTOR & (cer4816D.ipt) & \\
\hline $\begin{array}{l}3.0 \\
1.0\end{array}$ & 0.0 & 2.0 & 0.0 & 2.0 & 2.0 & 1.0 \\
\hline 0.0 & 0.0 & 0.0 & & & & \\
\hline 0.0 & 0.0 & 0.0 & 0.0 & & & \\
\hline 0.0 & 72.0 & 1.0 & 1.0 & & & \\
\hline 5.0 & 1.0 & 16.0 & 1.0 & & & \\
\hline-4.8 & 1.0 & 1.0 & 300.0 & & & \\
\hline 2.0 & 2.0 & 2.0 & 16.5 & & & \\
\hline 2.0 & 12.0 & 1.0 & 180 & 30.0 & 1.0 & \\
\hline 0.0 & 0.0 & 1.0 & 10.0 & 11.0 & 13.0 & \\
\hline 2.0 & 3.0 & 0.25 & 2.90 & 4.12 & & \\
\hline $\begin{array}{l}0.0 \\
12.0\end{array}$ & 0.0 & 0.0 & 0.0 & 0.0 & 0.0 & \\
\hline 2.90 & 3.50 & 4.12 & 4.95 & 6.40 & 7.69 & 9.41 \\
\hline 10.45 & 12.41 & 13.41 & 15.41 & 16.41 & & \\
\hline 0.600 & 1.447 & 2.418 & 2.333 & 2.185 & 2.057 & 1.883 \\
\hline 1.778 & 1.581 & 1.480 & 1.276 & 1.175 & & \\
\hline 12.0 & & & & & & \\
\hline 2.90 & 3.50 & 4.12 & 4.95 & 6.40 & 7.69 & 9.41 \\
\hline 10.45 & 12.41 & 13.41 & 15.41 & 16.41 & & \\
\hline 0.0 & -9.9 & -20.04 & -14.29 & -7.98 & -4.72 & -2.08 \\
\hline-1.12 & 0.02 & 0.48 & 1.35 & 1.78 & & \\
\hline 12.0 & & & & & & \\
\hline 2.90 & 3.50 & 4.12 & 4.95 & 6.40 & 7.69 & 9.41 \\
\hline 10.45 & 12.41 & 13.41 & 15.41 & 16.41 & & \\
\hline 0.0 & 0.0 & 0.0 & 0.0 & 0.0 & 0.0 & 0.0 \\
\hline 0.0 & 0.0 & 0.0 & 0.0 & 0.0 & & \\
\hline 11.0 & & & & & & \\
\hline 001.0 & 2.90 & 3.50 & & & & \\
\hline 001.0 & 3.50 & 4.12 & & & & \\
\hline 001.0 & 4.12 & 4.95 & & & & \\
\hline 001.0 & 4.95 & 6.40 & & & & \\
\hline 001.0 & 6.40 & 7.69 & & & & \\
\hline 001.0 & 7.69 & 9.41 & & & & \\
\hline 001.0 & 9.41 & 10.45 & & & & \\
\hline 001.0 & 10.45 & 12.41 & & & & \\
\hline 001.0 & 12.41 & 13.41 & & & & \\
\hline 001.0 & 13.41 & 15.41 & & & & \\
\hline 001.0 & 15.41 & 16.41 & & & & \\
\hline
\end{tabular}




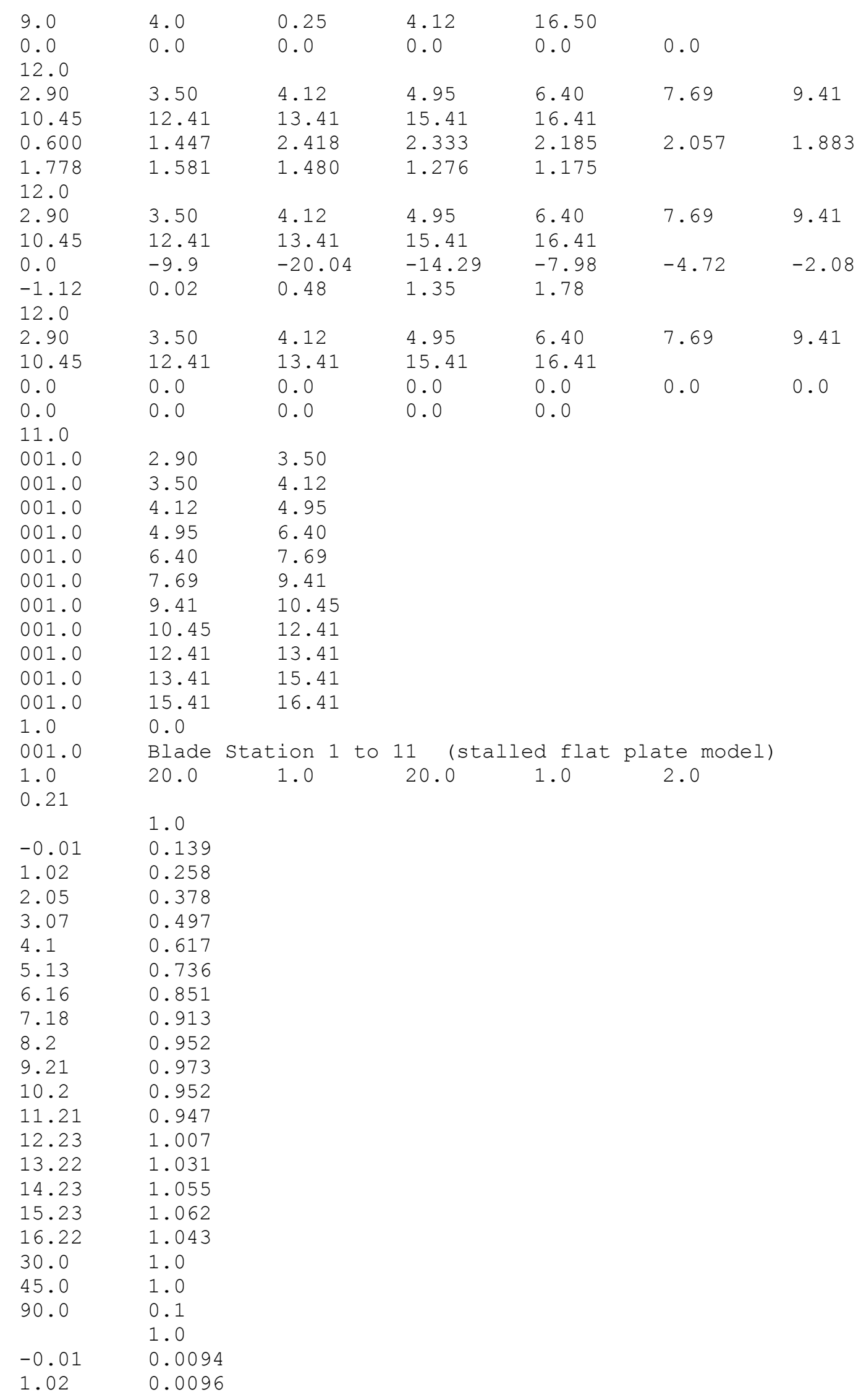




$\begin{array}{ll}2.05 & 0.0099 \\ 3.07 & 0.01 \\ 4.1 & 0.01 \\ 5.13 & 0.0097 \\ 6.16 & 0.0095 \\ 7.18 & 0.0127 \\ 8.2 & 0.0169 \\ 9.21 & 0.0247 \\ 10.2 & 0.0375 \\ 11.21 & 0.0725 \\ 12.23 & 0.0636 \\ 13.22 & 0.0703 \\ 14.23 & 0.0828 \\ 15.23 & 0.1081 \\ 16.22 & 0.1425 \\ 30.0 & 0.5 \\ 45.0 & 1.0 \\ 90.0 & 1.6 \\ -180.0 & 0.0 \\ 180.0 & 0.0\end{array}$




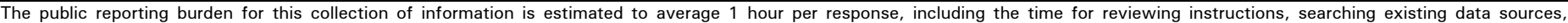

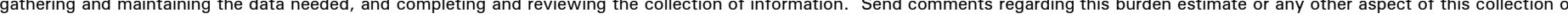

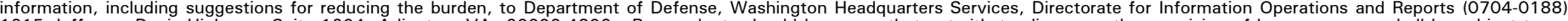

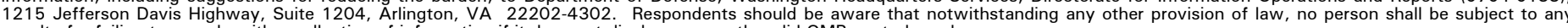
penalty for failing to comply with a collection of information if it does not display a currently valid OMB control number.

PLEASE DO NOT RETURN YOUR FORM TO THE ABOVE ADDRESS.

\section{\begin{tabular}{l|l} 
1. REPORT DATE $(D D-M M-Y Y Y Y)$ & 2. REPORT TYPE
\end{tabular} \\ 13-02-2004 \\ Technical Report}

4. TITLE AND SUBTITLE

Evaluation of RCAS Inflow Models for Wind Turbine Analysis
3. DATES COVERED (From - To)

a. CONTRACT NUMBER

5b. GRANT NUMBER

5c. PROGRAM ELEMENT NUMBER

5d. PROJECT NUMBER

Golden, CO 80401

9. SPONSORING/MONITORING AGENCY NAME(S) AND ADDRESS(ES)

10. SPONSOR/MONITOR'S ACRONYM(S)

11. SPONSOR/MONITOR'S REPORT NUMBER(S) 5e. TASK NUMBER

WER3 3110

5f. WORK UNIT NUMBER

6. AUTHOR(S)
J. Tangler, G. Bir

7. PERFORMING ORGANIZATION NAME(S) AND ADDRESS(ES)
National Renewable Energy Laboratory
1617 Cole Blvd.

6. AUTHOR(S)
J. Tangler, G. Bir

7. PERFORMING ORGANIZATION NAME(S)
National Renewable Energy Laboratory
1617 Cole Blvd.
Gen

6. AUTHOR(S)
J. Tangler, G. Bir

7. PERFORMING ORGANIZATION NAME(S)
National Renewable Energy Laboratory
1617 Cole Blyd.

\section{REPORT NUMBER}

NREL/TP-500-35109

12. DISTRIBUTION/AVAILABILITY STATEMENT

\section{SUPPLEMENTARY NOTES}

\section{ABSTRACT}

The finite element structural modeling in the Rotorcraft Comprehensive Analysis System (RCAS) provides a state-of-the-art approach to aeroelastic analysis. This, coupled with its ability to model all turbine components, results in a methodology that can simulate complex system interactions characteristic of large wind. In addition, RCAS is uniquely capable of modeling advanced control algorithms and the resulting dynamic responses.

15. SUBJECT TERMS

wind energy; wind turbines; RCAS; aeroelastic analysis

\begin{tabular}{|l|l|l|}
\hline 16. SECURITY CLASSIFICATION OF: \\
\hline a. REPORT & b. ABSTRACT & c. THIS PAGE \\
& & \\
\hline
\end{tabular}

\begin{tabular}{|l|l|l|}
\hline 17. LIMITATION OF & 18. NUMBER & 19 \\
ABSTRACT & OF \\
PAGES & & $19 b$
\end{tabular}

19a. NAME OF RESPONSIBLE PERSON

19b. TELEPHONE NUMBER (Include area code) 